\title{
Applications of gold nanoparticles in medicine and therapy
}

\begin{abstract}
The stability and dispersity of AuNMs in solution play a key role for the many applications. Most inorganic nanomaterials are not well dispersed in physiological buffers and require function-alization by thiols or surfactants to offer the stabilization forces. Furthermore, sufficient blood circulation time is critical for both imaging and in vivo drug delivery. Localized surface Plasmon resonance (LSPR) is one of the most significant features of AuNMs. The AuNMs as reporters have been broadly applied into lateral flow immunechromatographicalassay (LFICA) and enzyme-linked immunosorbent assay (ELISA), which is a well-established technology for analysis of the target analytes in food safety, clinical diagnosis, environmental monitoring, and medical science and so on. $\mathrm{Au}$ based nanomaterials (AuNMs) are known to possess many attractive features such as unique electrical, optical and catalytic properties as well as excellent biocompatibility. In this review, we summarize the current advancement on application of AuNMs in analytical sciences based on their local surface plasmon resonance, fluorescence and electrochemistry properties. AuNMs based imaging and therapy in biomolecules is explained. As one of the most reliable imaging modes, computed tomography (CT), X-ray and SERS imaging has been widely used owing to its high spatial and density resolution. We end the review by a discussion of the conjugation between gold nanoparticles with other kinds of nanoparticles such as other metals and carbon nano structures. Finally, future development in this research area is also prospected.
\end{abstract}

Volume 6 Issue 3 - 2018

Loutfy H Madkour

Chemistry Department, Faculty of Science, Al Baha University, Saudi Arabia

Correspondence: Loutfy H Madkour, Department of Chemistry, Faculty of Science, Baljarashi, Al Baha University, Al Baha, Saudi Arabia, Tel +966 533899075, Email loutfy_madkour@yahoo.com

Received: April 30, 2017| Published: May 08, 2018

\section{Introduction}

In 2017 Madkour $\mathrm{LH}^{1}$ published a vision for life sciences: interfaces between nano-electronic and biological systems in order to the prevention and treatment of disease in the human body. In this article, we review the applications of gold nanoparticles in medicine. Generally, the recognition elements, which are applied into colorimetric sensors based on AuNMs, are categorized as follows:

a. Antibodies or proteins modified AuNMs with immuno reaction,

b. Chemically-modified AuNMs with non-covalent bond recognition, and

c. Aptamers modified AuNMs with conformational change.

Furthermore, some novel techniques have been tried to improve the selectivity of colorimetric sensing, such as click-chemistry-based assay. Physics, chemistry and biology of Au based nanomaterials (AuNMs) have emerged as a broad and new sub discipline in the community of colloids and surfaces. The specific size and shape dependent physiochemical properties and remarkable bio/chemical inertness of AuNMs have made themselves the ideal candidates for both fundamental and technical study including crystal growth, electron-transfer mechanism, localized electro-magnetic theory, catalysis, DNA assay, bio-imaging and therapy, and so on. ${ }^{2}$ Among those rich properties, the optical characteristics originated from the giant electromagnetic field near the surface of AuNMs are particularly intriguing and thus broadly applied in analytical science, e.g. colorimetric assay, surface enhanced Raman spectroscopy (SERS) and surface Plasmon resonance (SPR) spectroscopy, as well as bio-imaging. Another interesting optical property of AuNMs is that fluorescence appears with their size shrinkage to below $2 \mathrm{~nm}$, which allows development of luminescence-based analysis. Also, electrochemical
(EC) sensors could be constructed based on the redox feature of $\mathrm{Au}$ NMs (Figure 1). Analytical science has been playing a primary role in our daily life, for instance, food safety control, ${ }^{3}$ biomedical diagnosis, ${ }^{4}$ medicolegal appraisement, ${ }^{5}$ anti-terrorism alert, ${ }^{6}$ and environmental pollution monitoring. ${ }^{7}$ Efficient detection technologies depend greatly on the analytical method in terms of analysis time, signal-to-noise $(\mathrm{S} / \mathrm{N})$ ratio, selectivity, and limit of detection (LOD). ${ }^{8}$ Therefore, a great of efforts should be paid to develop new analytical methods with the advantages of rapid determination, high analysis flux, satisfactory sensitivity and good robustness. Thanks to the above-mentioned optical and electrochemical features, many functionalized AuNMs have been successfully synthesized ${ }^{9-14}$ for improving performances of analytical techniques. (Figure 1)

\section{Small organic molecules and metal ions}

For the sensitive detection of small organic molecules, the AuNMs play an important role in improving sensitivity of the EC sensors. Especially, AuNMs composited with conducting polymers or carbon nanomaterials have been used to increase electro-chemical efficiency. It deserves noting that the decorated carbon nanomaterials with metal NPs are of special interest due to the integrated property of two components with better catalytic activity and enhanced electrical conductivity. ${ }^{15,16}$ Zhu et al. ${ }^{17}$ fabricated EC sensors by using spherical AuNPs combined with conducting polymer (poly-[2, 5-di(2- thienyl)-1H-pyrrole-1-(p-benzoic acid)]). ${ }^{17}$ To further improve electron transfer, synergistic effect of multiple nano=composite layers, namely, chitosan-AuNPs, graphene-AuNPs and multi-walled carbon nanotubes (MWCNTs)-cobalt phthalocyanine, was exploited by Sun et al. ${ }^{18}$ Roushani et al. ${ }^{19}$ fabricated a sensitive and selective EC aptasensorfor detection of cocaine based on the conformational change of the aptamer-functionalized AuNPs onto CNTs-based nanocomposite. Detection of organic small molecules and metal 
ions is very important for controlling food quality, environmental protection and antiterrorism. In order to realize detection of trace amount of analytes at extremely low concentrations, various efforts for increasing the sensitivity of SPR biosensors have been paid. Most of these methods are based on changing the refractive index at the sensor surfaces by means of a variety of (bio) chemical or NP agents, which are captured by the sensor surfaces subsequent to the binding of the target analyte to the primary biorecognition elements. These methods include the use of secondary and tertiary antibodies, antibodies labeled with enzymes, and dielectric or metallic NPs. ${ }^{20}$ In particular; AuNMs of diameters ranging from 5 to $40 \mathrm{~nm}$ have been widely used to enhance the response of SPR biosensors..$^{21,22}$

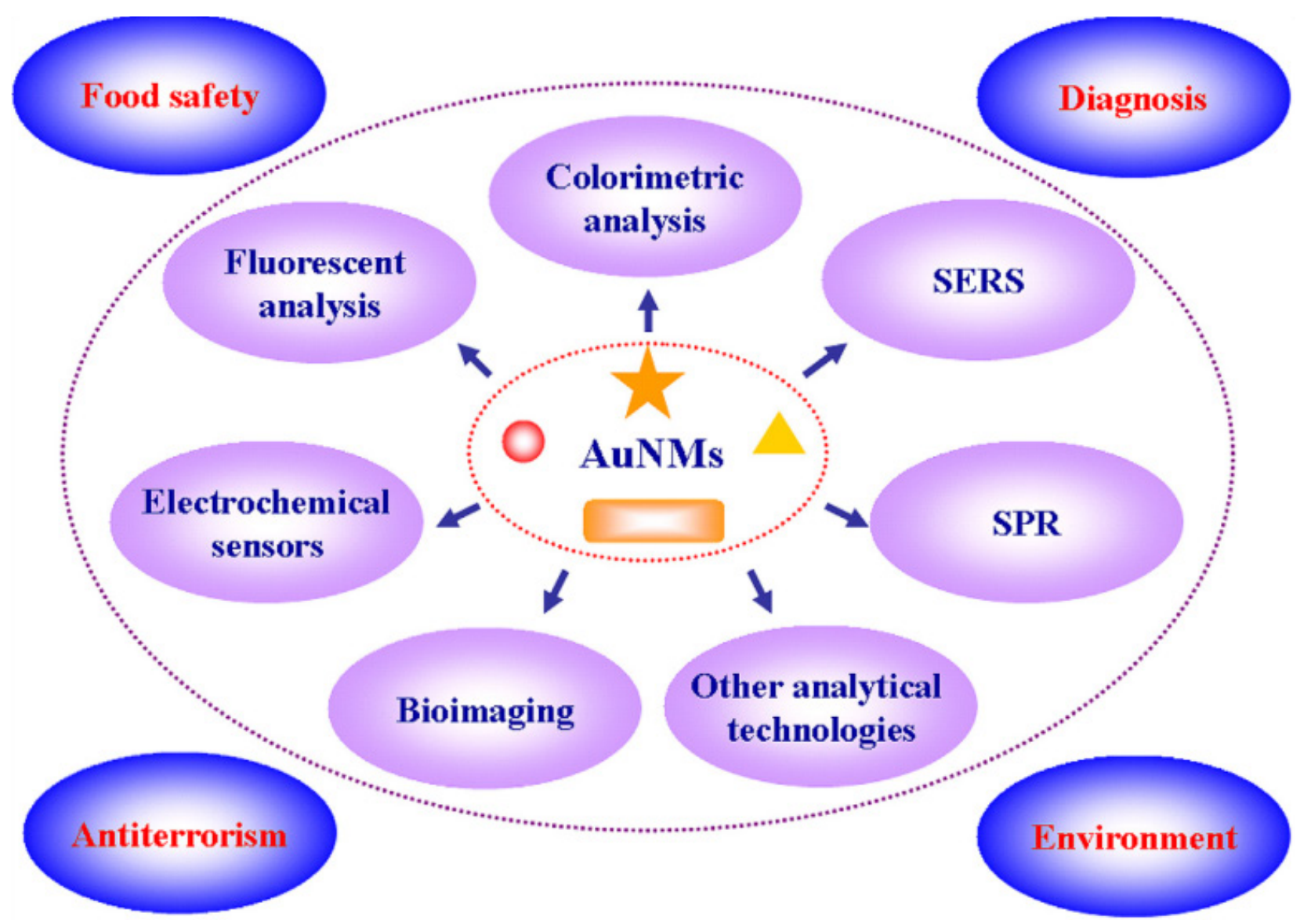

Figure I Application of AuNMs in analytical science.

\section{Metal ions}

Some EC sensor methods based on AuNMs have been developed for detection of heavy metal ions. For examples, Shen et al. ${ }^{23}$ proposed an EC DNAzyme sensor based on AuNPs for sensitive and selective detection of $\mathrm{Pb}^{2+}$. A label-free EC sensor was developed for the highly sensitive and selective detection of $\mathrm{Hg}^{2+}$ by Tang et al. ${ }^{24}$ In their study, the catalytic $\mathrm{HAuCl}_{4} / \mathrm{NH}_{2} \mathrm{OH}$ reaction was utilized for formation of AuNPs as signal reporter after capturing $\mathrm{Hg}^{2+}$ on the modified electrode surface through the specific thymine- $\mathrm{Hg}^{2+}$-thymine $\left(\mathrm{T}-\mathrm{Hg}^{2+}-\mathrm{T}\right)$ coordination. Compared with the traditional metal NPs-based method, this sensor avoided the labeling of the DNA probe with NP tags, and only one unlabeled T-rich DNA sequence was needed, which greatly reduced the cost and simplified the sensing procedure. Thus, a LOD as low as $0.06 \mathrm{nM}$ could be obtained for $\mathrm{Hg}^{2+}$. Furthermore, in 2016,
Wang et al. ${ }^{25}$ presented a sensitive, selective and reusable EC biosensor for determination of $\mathrm{Hg}^{2+}$ based on thymine modified AuNPs/reduced graphene oxide nanocomposites (Figure 2). ${ }^{25}$ Notably, the developed EC sensors afforded excellent selectivity for $\mathrm{Hg}^{2+}$ against other heavy metal ions including $\mathrm{Zn}^{2+}, \mathrm{Cd}^{2+}, \mathrm{Pb}^{2+}, \mathrm{Cu}^{2+}, \mathrm{Ni}^{2+}$ and $\mathrm{Co}^{2+}$. Wang et $\mathrm{al}^{26}$ prepared an EC sensor based on graphene and AuNPs for detection of trace $\mathrm{Cu}^{2+}$ with the anodic stripping voltammetry analysis. ${ }^{26}$ Yang et al. ${ }^{27}$ constructed a highly sensitive electro-chemical DNA biosensor made of polyaniline (PANI) and AuNPs nanocomposite (AuNPs@ PANI) for detection of trace concentration of $\mathrm{Ag}^{+} \cdot{ }^{27}$ In the presence of $\mathrm{Ag}^{+}$, with interaction of cytosine- $\mathrm{Ag}^{+}$-cytosine $\left(\mathrm{C}-\mathrm{Ag}^{+}-\mathrm{C}\right)$, cytosinerich DNA sequence immobilized onto the surface of AuNPs@PANI had a self-hybridization and then formed a duplex-like structure. The prepared EC sensors could detect $\mathrm{Ag}^{+}$at a wide linear range of $0.01-$ $100 \mathrm{nM}$ with a LOD of 10pM (Figure 2). 


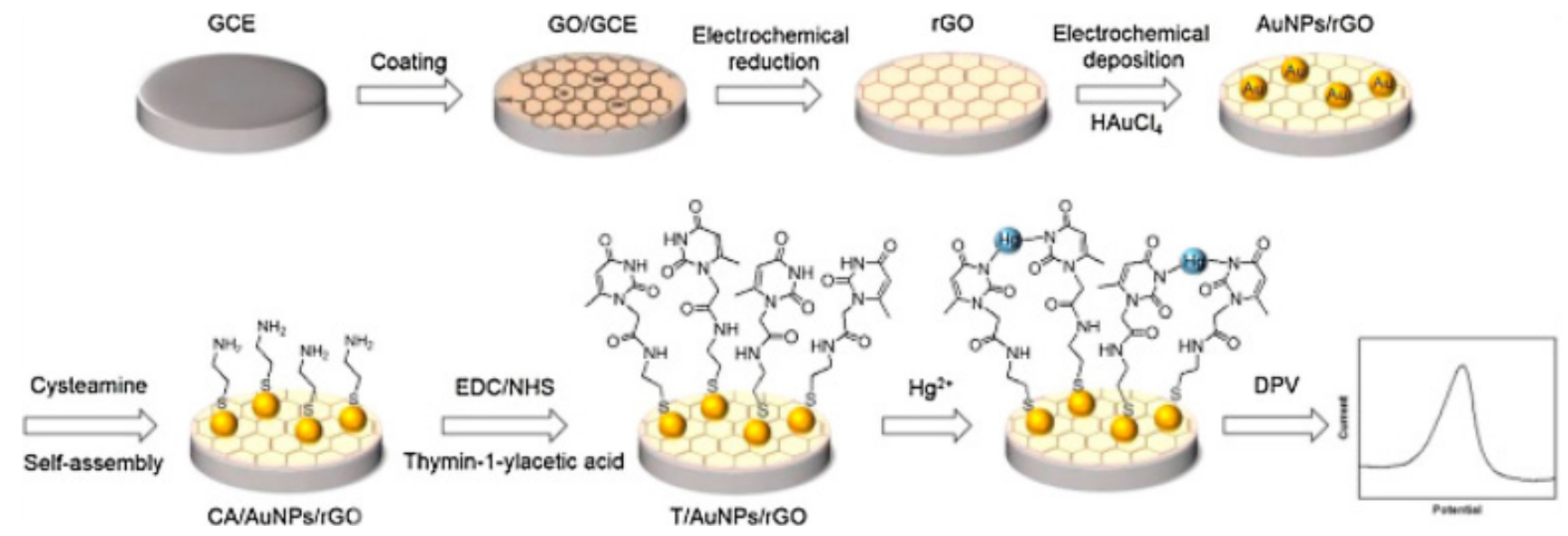

Figure 2 Scheme of thymidine functionalized biosensor for $\mathrm{Hg}^{2+}$ detection. ${ }^{25}$

\section{Synthesis of AuNMs}

Traditionally, the tri-sodium citrate-based thermal reduction has been broadly recognized as a universal approach for preparing spherical Au nanoparticles (AuNPs) of varied sizes ranging from 15 to $147 \mathrm{~nm}$ possessing strong SPR signals..$^{28}$ Although this preparation method simply involves controlling the ratios of citrate to $\mathrm{Au}$ ions, ${ }^{29}$ the reaction often needs to be carried out on a relatively large scale at the temperature of boiling water and lacks the reproducible preparation of uniform AuNPs with rather large sizes. After the breakthroughs reported by Schmid ${ }^{30,31}$ and Brust et al. ${ }^{32}$, a variety of methods have been developed to synthesize AuNMs, and many excellent reviews ${ }^{33}$ are available now. In general, the reported synthesis methods of spherical AuNMs can be categorized as followed:

a. Citrate reduction;

b. Brust-Schiffrin phase transfer synthesis;

c. Other sulfur-containing ligands method;

d. Soft templates including micro emulsion, reversed Micelles, surfactants, membranes and polyelectrolytes;

e. Seed-mediated growth;

f. Physical methods. ${ }^{34}$

In addition, non-spherical nanoparticles (NPs) such as Au nanorods (AuNRs), ${ }^{35} \mathrm{Au}$ nanocages, ${ }^{36} \mathrm{Au}$ cubes,${ }^{37} \mathrm{Au}$ triangular prisms, ${ }^{38}$ as well as other unconventional structures like hollow tubes and even branched nanocrystals ${ }^{39}$ have attracted significant research attention in the past two decades, since the properties of AuNMs are known to be strongly dependent on the size and shape of the particle. ${ }^{40}$ Recently, a lot of excellent reviews have summarized how to achieve shape and size-defined AuNMs with different techniques including solve thermal synthesis, seed-mediated growth, homogeneous nucleation and electrochemical method. ${ }^{41,42}$ In this review, we mainly introduce the latest and important advancement of synthesis of the AuNMs, especially on how to control the morphology of AuNMs. A great of efforts have been paid to achieve high-quality and shape-controlled
AuNMs for the purpose of highly-sensitive analysis. Unfortunately, most of the synthetic methods are empirical, and understanding their growth mechanism remains a challenging task. Interestingly, introduction of $\mathrm{Ag}^{+}$in growth solutions significantly improves the yield of AuNRs, and Yang et al ${ }^{43}$ prepared AuNRs with controlled aspect ratio by using photochemistry in the presence of $\mathrm{Ag}^{+} .{ }^{43}$ This process itself was highly promising for producing uniform AuNRs, and more importantly it was useful in deciphering the growth mechanism of anisotropic AuNPs due to its simplicity and the relatively slow growth rate of the AuNRs.

Later, a versatile seed-mediated growth method was reported for selective synthesis of single crystalline rhombic dodecahedral, octahedral, and cubic AuNMs. ${ }^{44}$ These results might provide a basis for gaining mechanistic insight into the growth of shape-and structure-controlled noble metal nanomaterials. An even more complex sample is nanostar, which is characteristic of uneven arms with different lengths. To fully implement the advantageous property of Au nanostars, a precise control over their symmetry and uniformity is highly desired. In 2015, Niu et al. ${ }^{45}$ obtained Au nanostars with excellent symmetry control by using a solution-phase method in high yield and good monodispersity. ${ }^{45}$ In their study, icosahedral seeds were used to dictate the growth of the evenly distributed arms in an $\mathrm{I}_{h}$ symmetric manner. Benefiting from their high symmetry, the $\mathrm{Au}$ nanostars exhibited much better single-particle SERS performance compared with asymmetric Au nanostars, in terms of both intensity and reproducibility. Apart from large-sized Au particles, Au nanoclusters (AuNCs) with the sizes of less than $2 \mathrm{~nm}$ formed in dendrimers displays visible luminescence of high quantum yield (QY). ${ }^{46}$ Ying et al. ${ }^{47}$ reported a simple, one-pot and "green" synthetic route, based on the reduction and stabilization capability of bovine serum albumin (BSA) proteins. The AuNCs prepared at the physiological temperature $\left(37^{\circ} \mathrm{C}\right)$ showed red emission with a QY of $\sim 6 \%{ }^{47}$

In order to further control the luminescence property, synthesis of atomically precise AuNCs has ignited numerous research efforts. ${ }^{48,49}$ In 2014, Yu et al..$^{50}$ synthesized $\mathrm{Au}_{22}$ (glutathione) ${ }_{18}$ clusters of the emission peak at $\sim 665 \mathrm{~nm}$ with a QY of $\sim 8 \%$. Upon comparison of their luminescence properties with those of $\mathrm{Au}$ (I)-thiolate complexes, 
the enhanced luminescence of $\mathrm{Au}_{22}(\mathrm{SG})_{18}$ was ascribed to aggregationinduced emission. Nevertheless, the origin of emission in AuNCs still keeps unclear, posing a major hurdle for development of highly luminescent clusters. In 2015, Pyo et al. ${ }^{51}$ prepared $\mathrm{Au}_{22}$ (glutathione) ${ }_{18}$ clusters with a luminescence QY greater than $60 \%$ by rigidifying their $\mathrm{Au}$ shell with tetraoctylammonium cations. ${ }^{51}$ This study presents an effective strategy to enhance the luminescence efficiencies of AuNCs by optimizing the shell structure. In order to precisely tune the size and shape of AuNMs, DNA is recently adopted as a powerful programmable tool to realize above goals. Lu's group systematically summarized morphology controlling, spatial positioning and dynamic assembly of AuNMs with DNA as a powerful tool..$^{52}$ In some details, their research results demonstrated that DNA could be used to control the morphologies of AuNMs during seed-mediated growth. ${ }^{53}$ They also developed a novel method based on DNA-encoded tuning to achieve AuNMs from AuNR seeds with controlled geometric and plasmonic properties. ${ }^{54}$ Furthermore, they suggested that the DNA molecules played important roles via influencing diffusion of the $\mathrm{Au}$ precursors to the seed, and therefore the AuNMs growth might be modulated through difference in DNA desorption, density and mobility on the seed surfaces. ${ }^{55}$

These insights into the mechanism of DNA-guided AuNMs synthesis not only provide deep understanding of the interactions between the DNA and nanomaterials but also allow better control of the shapes and surface properties of many nanomaterials. Ye et al. ${ }^{56}$ designed a millifluidic reactor to synthesize a wide variety of AuNMs at high concentrations. ${ }^{56}$ The synthesized method based on millifluidic reactors inspired other researchers to use the analogous reactors for possible mechanism and application study. Recently, Krishna et al. ${ }^{57}$ demonstrated adoption of a simple millifluidic chip for in situ analysis of morphology and dimension-controlled growth of Aunano and micro-structures with a time resolution of $5 \mathrm{~ms}$ (Figure 3). ${ }^{57}$ See from Figure 3, gold structures with $\sim 3 \mu \mathrm{m}$ in diameter that had corrugated metal-like shapes were formed after a time interval of $5 \mathrm{~h}$ (Figure $3 \mathrm{D})$. When the time of flow was increased to $9 \mathrm{~h}$, these structures further transformed into micro-hemispherical ones (Figure 3C). This study offers a feasible way to obtain dimension-and morphologycontrolled AuNMs in a high yield (Figure 3). Noteworthy, some novel synthesis strategies, such as biosynthesis, ${ }^{58,59} 2 \mathrm{D}$ immiscible oil/water interface, ${ }^{60}$ levitated leidenfrost drop, ${ }^{61}$ sunlight-induced synthesis ${ }^{62}$ and so on, have been reported, and such green and economic synthesis methods for preparation of uniform and controlled AuNMs provide the opportunity for development of low-cost and sustainable analytical techniques. However, the above technologies need to be further improved and standardized.

(a)

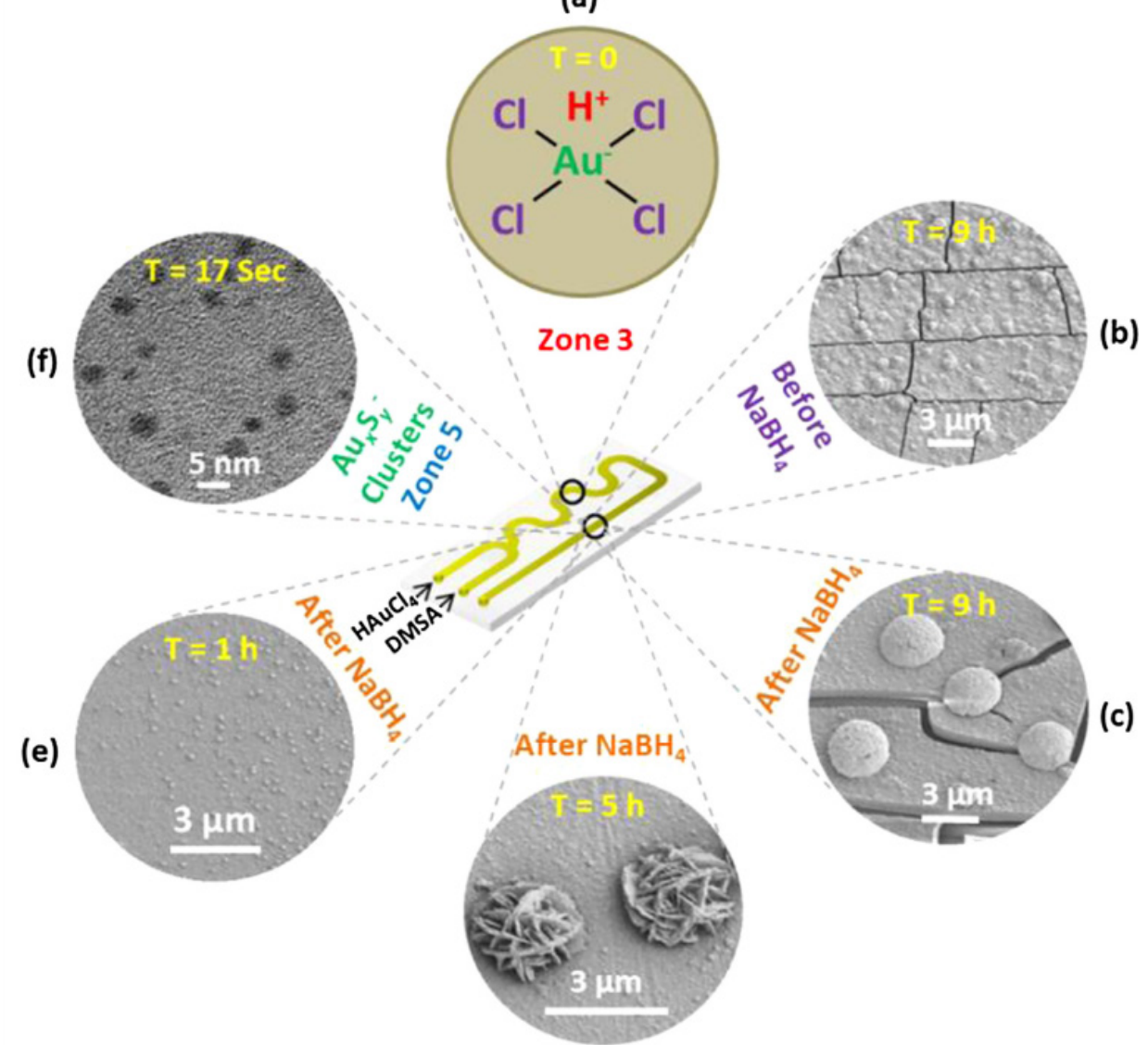

(d)

Figure 3 Scheme showing different stages of spatially (and time) resolved growth process of AuNMs within millifluidic chip. ${ }^{57}$ 


\section{Surface modification of AuNMs}

Owing to the large surface to volume ratio, modification and functionalization of AuNMs surfaces with biomolecules, DNA or chemicals becomes imperative for their application in analytical science. It is well known that free AuNMs have high surface energy and tend to aggregate and fuse. As a result, the intriguing properties observed for the AuNPs would disappear, and the difficulty arises for long term storage, processing, and applications. Therefore, great efforts have been devoted to develop novel strategies to stabilize AuNMs, ${ }^{63}$ and the most common approach is to coat AuNMs with either organic or inorganic shells. These shells not only endow AuNPs with high stability but also offer them additional functionalities. As an example, in addition to good stability and biocompatibility, the mesoporous silica shells that are currently broadly used have high surface area and tunable pore size and volume, which can accommodate analytes and drug molecules. ${ }^{64}$

\section{Biomolecules (DNA, protein and cell)}

AuNMs have been widely used in SERS based immunoassays of biomolecules such as DNA, protein and cell. However, challenges still remain with amplification of SERS signals due to the extremely small cross-section of Raman scattering. ${ }^{65}$ Yan et al. intro-duced a new strategy based on nano rolling-circle amplification (nanoRCA) and nano hyper branched rolling circle amplification(nanoHRCA) to increase "hot spot" groups for protein microarrays. ${ }^{66} \mathrm{Li}$ et al.${ }^{67}$ constructed a SERS immune sensor for detection of biomarkers ${ }^{67}$ In their study, the capture antibody was immobilized on the Au triangle nanoarray chip, while the detection antibody was conjugated with the SERS probe (Au@Ramanreporter@SiO, sandwich NP). The antigen (analyte) was sandwiched between the capture antibody and the detection antibody. Under light excitation, a lot of "hot spots" were created between the Au triangles in nanoarray, and thus the developed SERS immunosensor could be used for sensitive biomarker detection. Luo et al ${ }^{68}$ reported a facile immunoassay for porcine circovirus type 2 (PCV2) based on SERS using multi-branched AuNPs (mbAuNPs) as substrates. The mb-AuNPs in the immunosensor act as Raman reporters, which were prepared via Tris base-induced reduction and subsequent reaction with $p$-mercaptobenzoic acid The modified mb-AuNPs were covalently conjugated to the monoclonal antibody $(\mathrm{McAb})$ against the PCV2 cap protein to form SERS immuno nanoprobes. These were captured in a microtiterplate via an immuno reaction in the presence of target antigens. Compared to conventional detecting methods such as those based on PCR, the method was demonstrated to be rapid, facile and very sensitive. Tang et al. developed a simple and reproducible SERS chip for highly sensitive and selective screening of active ricin in complex matrices. ${ }^{69}$ They fabricated single strand oligodeoxynucleotides modified AuNPs in order to amplify recognition and reaction by virtue of their cooperative binding property. To improve sensitivity of this method, a Ag nanoshell was deposited on post-reacted poly (21dA)-AuNPs, which lowered LOD to $8.9 \mathrm{ng} \mathrm{mL}^{-1}$.

The utilization of SERS-based techniques in targeting specific DNA and RNA sequences is generally realized by combination with AuNMs and Raman-active molecules. ${ }^{70,71}$ Wang et al. ${ }^{72}$ raised a concept to use enzyme controlled plasmonic coupling as SERS nanosensors for DNA demethylation. ${ }^{72}$ The nanosensors were constructed by decorating AuNPs with Raman reporters and hemimethylated DNA probes. The enzymatic degradation of DNA substrate probes was utilized to induce aggregation of AuNPs, so the reproducible and sensitive SERS signals were achieved from biological recognition events. Development of SERS-barcoded particles, which contain multiple Raman-active molecules and are insensitive to photo bleaching, is particularly intriguing for high-throughput and multiplex DNA screening. ${ }^{73}$ Recently, Chenet al. ${ }^{74}$ prepared a SERS-barcoded nanosensor to detect Bacillusthuringiensis $(\mathrm{Bt})$ gene transformed rice expressing insecticidal proteins. ${ }^{74}$ The barcoded sensor was designed by encapsulation of AuNPs with silica and conjugation of oligonucleotide strands for targeting DNA strands. The transition between the cry1A (b) and cry1A(c) fusion gene sequence was used to construct a specific SERS-based detection method with a LOD of $0.1 \mathrm{pg} / \mathrm{mL}$. In order to build the determination models to screen transgene, a series mixture of Bt rice and normal rice were prepared for SERS assay, and the LOD was $0.1 \%(\mathrm{w} / \mathrm{w})$ was explored for transgenic Bt rice relative to normal rice. The sensitivity and accuracy of the SERS-based assay were comparable with real-time PCR. Recently, Ye et al ${ }^{75}$ proposed an asymmetric signal amplification method for simultaneously detecting multiple biomarkers with significantly different levels. ${ }^{75}$ The biobarcode consisted of a large number of signals DNA (Cy3-DNA for Cy3-bio barcode or Rox-DNA for Rox-biobarcode) and capture DNA immobilized onto the AuNPs. The AuNPs simultaneously act both as a Raman-signal-enhancing substrate and a Raman signal carrier. Using these bi-functional probes, a linear amplification mode was obtained with high-concentration markers, whereas quadratic amplification mode was responsible to low-concentration markers.

Detection of circulating tumor cells (CTCs) in the blood of cancer patients is significant for early cancer diagnosis, cancer prognosis, evaluation of the treatment effect of chemotherapy drugs, and choice of cancer treatment options. ${ }^{76}$ In 2011, Wang et al. ${ }^{77}$ introduced SERS technology for direct detection of target CTCs in human peripheral blood. The LOD of this method ranged from 5 to $50 \mathrm{CTCs}$ in $1 \mathrm{~mL}$ of blood. ${ }^{77}$ Recently, Wu et al. ${ }^{78}$ fabricated SERSNPs for direct detection of CTCs in the blood with excellent specificity and high sensitivity. ${ }^{78}$ The AuNPs were encoded with a Raman reporter molecule, 4-mercaptobenzoic acid (4-MBA), and then functionalized with reductive bovine serum albumin (rBSA) to stabilize the 4-MBA-encoded AuNPs (AuNP-MBA) and decrease the nonspecific interaction with blood cells. Then AuNP-MBA-rBSA-FA composite NPs were constructed with a targeted ligand folic acid (FA) (Figure 4A) (Figure 4B). The FA on the surface of AuNP-MBA-rBSA-FA NPs was recognized by CTCs, which over expressed folate receptor alpha $(F R \alpha)$. The protection layer of rBSA was much thinner than that of the reported PEG, resulting in a stronger SERS signal (Figure 4C). (Figure 4) For detection of the biological macromolecules such as bacteria pathogen and enzyme, SPR with the advantage of label free provides a powerful platform. ${ }^{79,80}$ There are many successful examples of SPR sensing as a medical diagnostic tool, which have been reported for biomarkers, pathogen detection and hormone analysis with high sensitivity. For example, Mc Phillips et al. ${ }^{81}$ employed aligned Au nanotube arrays to strengthen performance of refractive index sensors in biomolecular binding reactions. ${ }^{81}$ Generally, sandwich ${ }^{82}$ and competitive or inhibition assay ${ }^{83}$ are two major detection approaches in SPR biosensor. In 2012, Fernandez et al. ${ }^{84}$ demonstrated that AuNMs combined with sandwich assays could improve detection sensitivity and selectivity of protein detection. ${ }^{84} \mathrm{Jung}$ et al. ${ }^{85}$ introduced a strategy for enhancement of SPR signals by adopting AuNPs and a $\mathrm{SiO}_{2}$ layer on a $\mathrm{Au}$ surface. The modified surfaces showed significant changes 
in SPR signal when biomolecules were attached to the surface as compared with an unmodified Au surface. The LOD of AuNPs immobilized on a SPR chip was $0.1 \mathrm{ngmL}^{-1}$ for the prostate specific antigen (PSA), a cancer marker. The enhanced shift of the absorption curve resulted from coupling of the surface and particle plasmon by the $\mathrm{SiO}_{2}$ layer and the AuNPs on the gold surface. Now, there were a lot of reports of SPR sensors based on spherical AuNMs for detection of biomolecules. ${ }^{86,87}$ The anti-E.coliO157:H7 polyclonal antibodies (pAb) were labeled with AuNPs, which were used as secondary antibodies. The E.coli O157:H7 was detected using direct assay and enhancing sandwich assay based on the two channels SPR biosensor. By introducing AuNPs-PAb compound, the LOD was determined to be $10 \mathrm{cfu} / \mathrm{mL}$. The sensitivity was 100 times higher than that of direct detection. ${ }^{88}$ Compared with spherical AuNMs, AuNRs labels are more favorable for the Au-amplified SPR biosensors because of the tunable longitudinal plasmonic peak enables an effective plasmonic coupling between sensing film and NPs. Law et al. ${ }^{89}$ revealed the potential of applying this "perfectly matched" nanotag in a well-established SPR sensing system and immunoassay. Through detection of tumor necrosis factor alpha antigen, 40-fold sensitivity enhancement using wavelength-matched AuNRs was observed ${ }^{89}$ Recently, Xiang et al. ${ }^{90}$ developed a SPR DNA biosensor array based on target-primed rolling circle amplification (RCA) for isothermal and rapid detection of two pathogenic mycobacteria, mycobacterium tuberculosis complex (MTBC) and mycobacterium avium complex (MAC) (Figure 5). AuNPs were directly assembled on to the surface of the sensor chip via hexanedithiol (HDT) for enhancement of sensitivity as a labelfree detection system. Experimental results showed that the signal enhancement by the target-primed RCA together with AuNPsembedded surface caused at least 10 -fold increased sensitivity as compared with conventional RCA on bare SPR chip method (Figure $5)$.

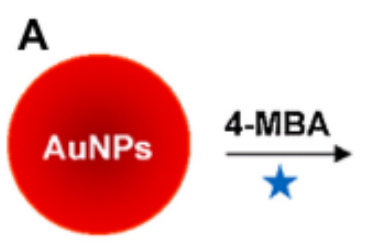

B
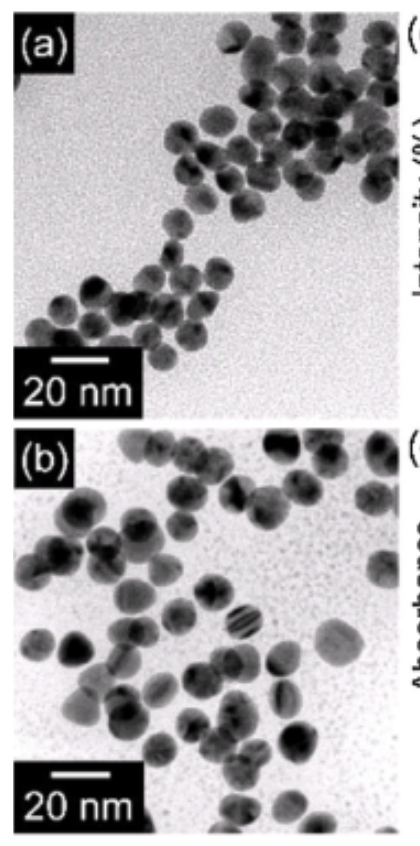
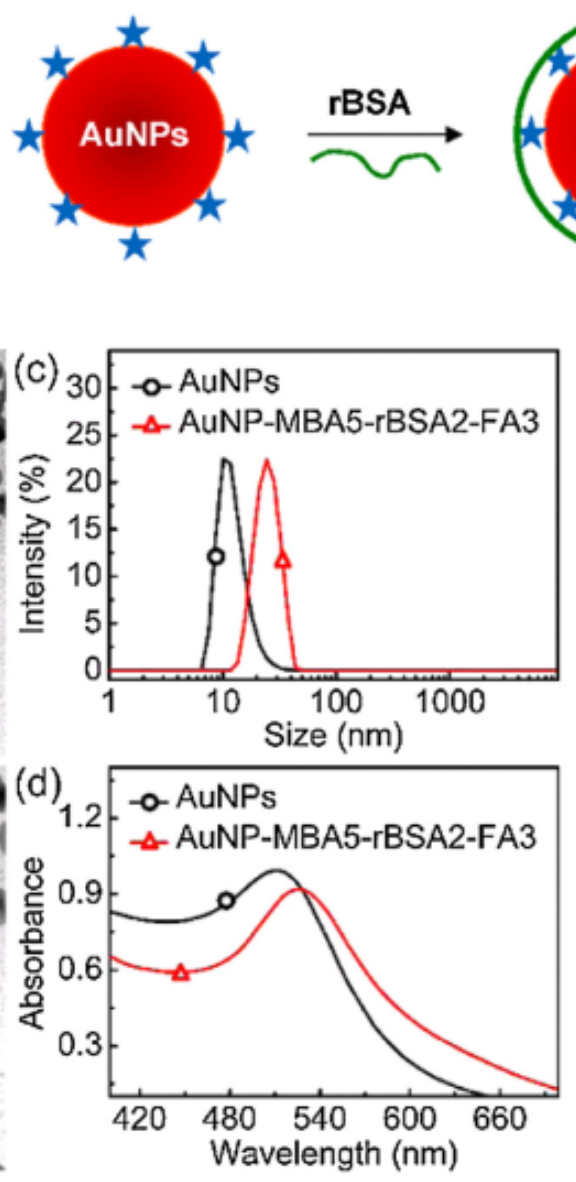
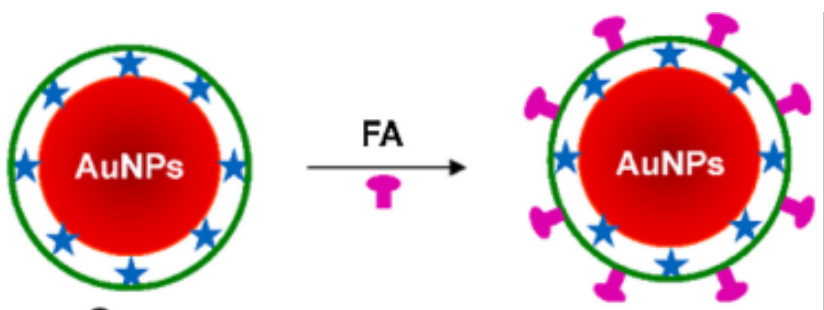

C

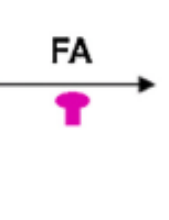

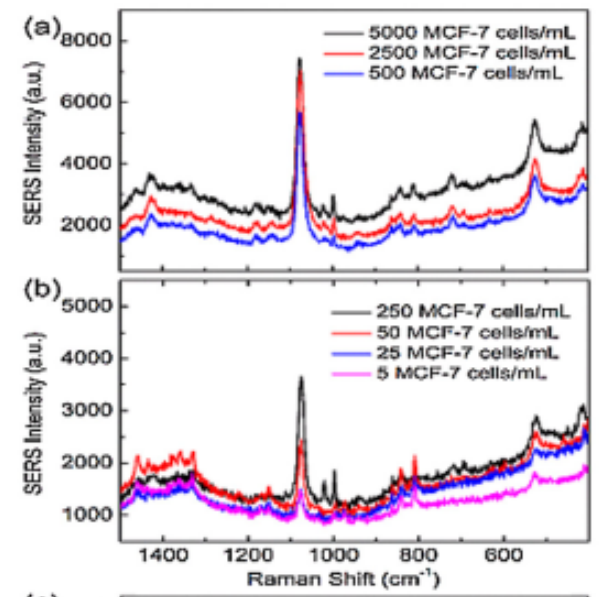

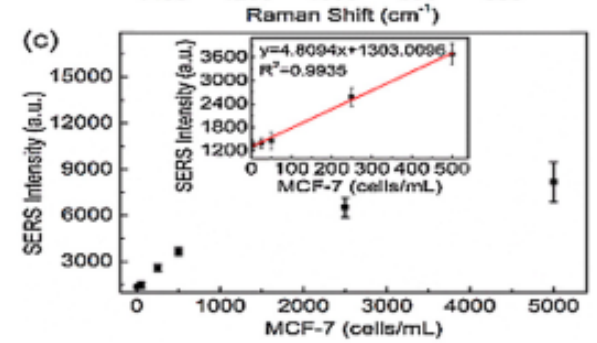

Figure 4 (A) Schematic Illustration for the Design of SERS Nanoparticles. (B) Characterization of the AuNPs (control) and SERS nanoparticles. (a) TEM images of AuNPs. (b) AuNP-MBA5-rBSA2-FA3. (c) Size distributions of AuNPs and AuNP-MBA5-rBSA2-FA3 in Milli-Q water at room temperature. (d) UV-vis spectra of AuNPs and AuNP-MBA5-rBSA2-FA3. (C) Detection sensitivity of the AuNP-MBA5-rBSA2-FA3 nanoparticles for MCF-7 cells in the rabbit blood. ${ }^{78}$ 
A
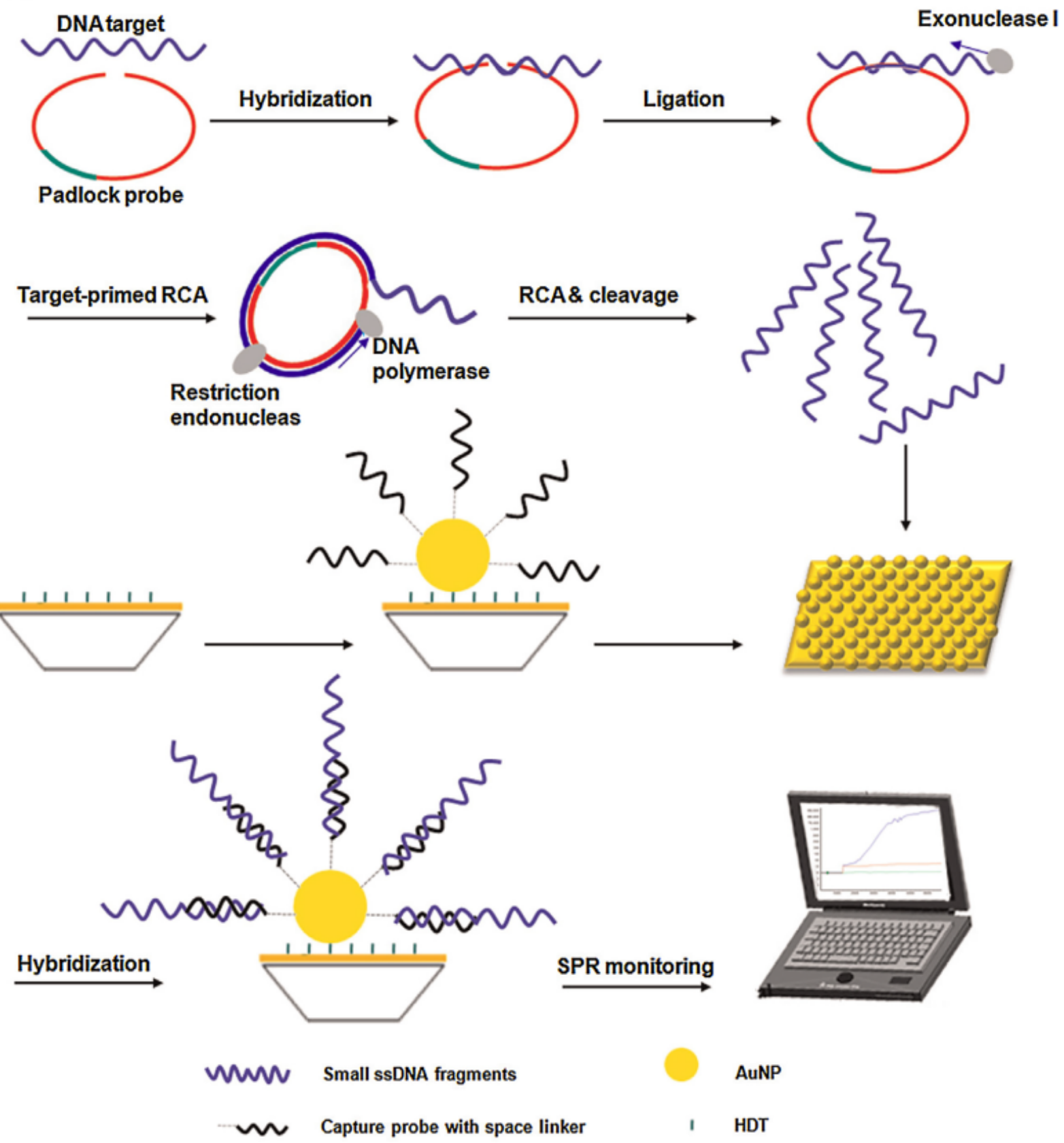

B

$$
5^{\prime}=\text { Ta code } \ldots \text { c............. Tb }
$$

Figure 5 (A) Principle of target-primed RCA-cleavage reaction-based AuNP-embedded SPR assay. (B) Mode of the padlock probe. Ta and Tb are asymmetric target complementary regions in padlock probe. Each padlock probe contains a unique code sequence for multiplex hybridization. The dotted line represents the bases in the linker sequence..$^{00}$ 
Besides, the chemical vapor deposition (CVD) method has been adopted by Ruoff's group for large scale preparation of highquality graphene sheets. ${ }^{91}$ The strategy based on AuNMs combined with graphene sheets could improve the conductivity of SPR. ${ }^{92}$ In 2013, Zhang et al. ${ }^{93}$ employed Au-graphene oxide composite inthe wavelength modulation SPR biosensor for rabbit $\operatorname{IgG}$ detection. ${ }^{93}$ In their study, the staphylococcal protein A (SPA) modified Augraphene oxide composite was directly immobilized onto SPR chips without any additional chemical treatment. The biosensor with the SPA modified Au-graphene oxide composite as the enhanced sensing platform exhibited a satisfactory response to rabbit IgG. The LOD obtained with the composite was 16 times lower than that obtained with the SPA modified chip. This study provided a simple and effective approach for fabrication of sensitive SPR immunosensors and extended the application of the Au-graphene oxide composite in immunoassays. Molecularly imprinted polymers (MIPs) have become a competitive tool in the field of molecular recognition, owing to their low cost, physical robustness, thermal stability and easy preparation over biological receptors and other functionalized materials. ${ }^{3}$ High surface-to-volume ratio makes AuNPs a competitive candidate as matrix material for novel nano sized MIPs. Xie et al. showed a surface molecular self-assembly strategy for MIP in electro polymerized aminothiophenol (ATP) membranes at the AuNPmodified glass carbon electrode for electrochemical detection of pesticidechlorpyrifos. ${ }^{94} \mathrm{Yu}$ et al..$^{95}$ constructed an EC sensor for determination of dopamine based on core-shell composite of AuNPs and $\mathrm{SiO}_{2} \mathrm{MIPs}$ through sol- gel technique. ${ }^{95}$ Similarly, Xue et al. ${ }^{96}$ prepared a highly sensitive and selective biomimetic EC sensor for amperometric detection of trace dopamine (DA) in human serums by AuNPs doped MIPs (Figure 6). The LOD of the proposed EC sensor for DA was $7.8 \mathrm{nmol} / \mathrm{L} .{ }^{96}$ Sun et al. ${ }^{97}$ presented an EC sensor for detection of 3-chloro-1, 2-propandiol (3-MCPD) that was usually used as surfactant. They fabricated an AuNPs-modified glassy carbon electrode coated with an MIP film via electro polymerization. The LOD reached $3.8 \times 10^{-18} \mathrm{~mol} / \mathrm{L}$. impressively, the sensor showed high sensitivity, good selectivity, excellent reproducibility and stability during the quantitative determination of 3-MCPD (Figure 6).

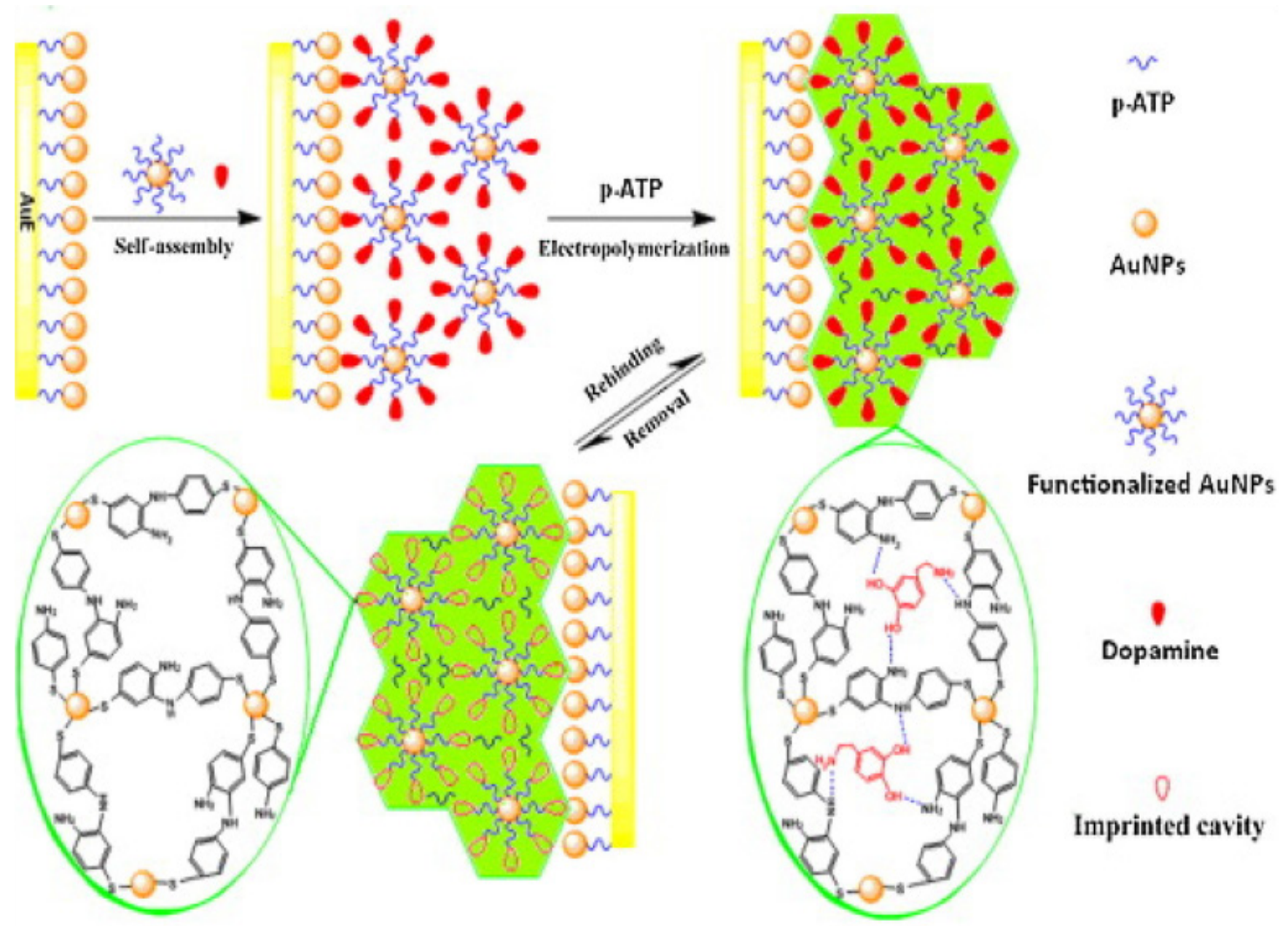

Figure 6 Scheme of preparation of AuNPs@MIES.96 
Ultrasensitive detection of biomolecules (especially for disease markers) is very important for early disease diagnosis. To realize ultrasensitive detection of biomolecules, development of novel and robust detection methods for signal amplification of EC sensors is essential. In past work, a lot of photo or electro-active indicators, such as ferrocene, ${ }^{98}$ methylene blue ${ }^{99}$ and enzymes, ${ }^{100}$ were immobilized onto AuNPs to realize the detection signals amplification. Zheng et al. ${ }^{101}$ prepared an ultrasensitive EC sensor based on networklike thiocyanuric acid/AuNPs for detection of thrombin. ${ }^{101}$ Zhao et al. ${ }^{102}$ fabricated an ultrasensitive and highly specific EC aptasensor for thrombin based on amplification of aptamer-AuNPs-horseradish peroxidase conjugates. ${ }^{102} \mathrm{He}$ et al. ${ }^{103}$ reported a label-free and sensitive signal-on electrochemical assay for MTase quantification and activity analysis using AuNP samplification. ${ }^{103}$ In 2016, an ultrasensitive DNA biosensor was developed through in situ labeling of electroactive melamine- $\mathrm{Cu}^{2+}$ complex $\left(\mathrm{Mel}-\mathrm{Cu}^{2+}\right)$ on the end of hairpin-like probe using AuNPs as the signal amplification platform by our group. ${ }^{104}$ In this work, the highly conductive AuNPs were applied as the loading platform of the Mel- $\mathrm{Cu}^{2+}$ tags. The proposed biosensor showed high selectivity, wide linear range and low LOD for target DNA. Except for the aforementioned amplification strategies, rolling circle amplification (RCA) is one of the most popular and important amplification strategies. Therefore, the dual amplification strategies combined RCA with DNA-AuNPs probe could tremendously improve the sensitivity of bacterial detection. Zhu et al. ${ }^{105}$ reported an electrochemical sensing strategy for ultrasensitive and rapid detection of Salmonella by combining the RCA with DNA-AuNPs probe. ${ }^{105}$ As shown in Figure 7, the target DNA could be specifically captured by probe 1 on the sensing interface. Then the circularization mixture was added to form a typical sandwich structure. In the presence of dNTPs and phi29 DNA polymerase, the RCA was initiated to produce micrometer-long single-strand DNA. Finally, the detection probe (DNA-AuNPs) recognized RCA product to produce enzymatic electrochemical signal. The developed method was successfully applied to detect Salmonella with lower LOD of
$6 \mathrm{CFUmL}^{-1}$ in real milk sample (Figure 7). Both of graphene based materials and AuNPs are the frequently used nanomaterials in the field of EC biosensors for detection of biomolecules due to their excellent electrical signal amplification and the versatile functionalization chemistry. Accordingly, hybrid nanomaterial sensors based on AuNPs distributing on the surface of graphene oxide (GO) or reduce GO (rGO) also attract much scientific interest. ${ }^{106,107}$ The most frequently used technique refers to reaction of $\mathrm{AuCl}_{3}$ with $\mathrm{GO}$ under reductive condition for in situ anchoring AuNPs to GO or rGO. This method, however, often lacks fine control over the size, uniformity and density of AuNPs on the GO sheets in the reaction process. ${ }^{108}$ AuNPs can be decorated to GO surface based on NH-Au binding ${ }^{109}$ or $\mathrm{SH}-\mathrm{Au}$ binding. ${ }^{110} \mathrm{DNA}^{111}$ and proteins ${ }^{112}$ have been also used as the molecular linkers between AuNPs and GO. These methods have greatly increased the uniformity and density of AuNPs. Liu et al. ${ }^{113}$ presented stable label-free EC sensor for detection of cardiac troponin-I (cTnI) in the early diagnosis of myocardial infarction based on AuNPs and GO nanocomposites. ${ }^{113}$ The EC sensor demonstrated good selectivity and high sensitivity against human-cTnI, and was capable of detecting cTnI at concentrations as low as $0.05 \mathrm{ngmL}^{-1}$, which was 100 times lower than that by conventional methods. Karaboga et al. ${ }^{114}$ described a simple and disposable immunosensor based on indium-tinoxide (ITO) sheets modified with AuNPs to sensitively analyze heat shock protein70 (HSP70), a potential biomarker that could be evaluated in diagnosis of some carcinomas. ${ }^{114}$ Wang et al. ${ }^{115}$ constructed an AuNPs/ polyaniline/chitosan-graphene sheets based electrochemical DNA sensor with functional hairpin probe for detection of $\mathrm{BCR} / \mathrm{ABL}$ fusion gene in chronic myel-ogenous leukemia. ${ }^{115}$ Recently, the interest in layered transition metal chalcogenides, especially $\mathrm{MoS}_{2}$, has been growing quickly because they share many impressive physicochemical properties of graphene. Su et al. have prepared an EC sensor based on $\mathrm{MoS}_{2}$ layer for sensitive detection of proteins. ${ }^{116}$ In subsequent work, they further fabricated a $\mathrm{MoS}_{2}$-based EC aptasensor for simultaneous detection of thrombin and adenosine triphosphate (ATP) based on AuNPs-MoS 2 nanocomposites. ${ }^{117}$

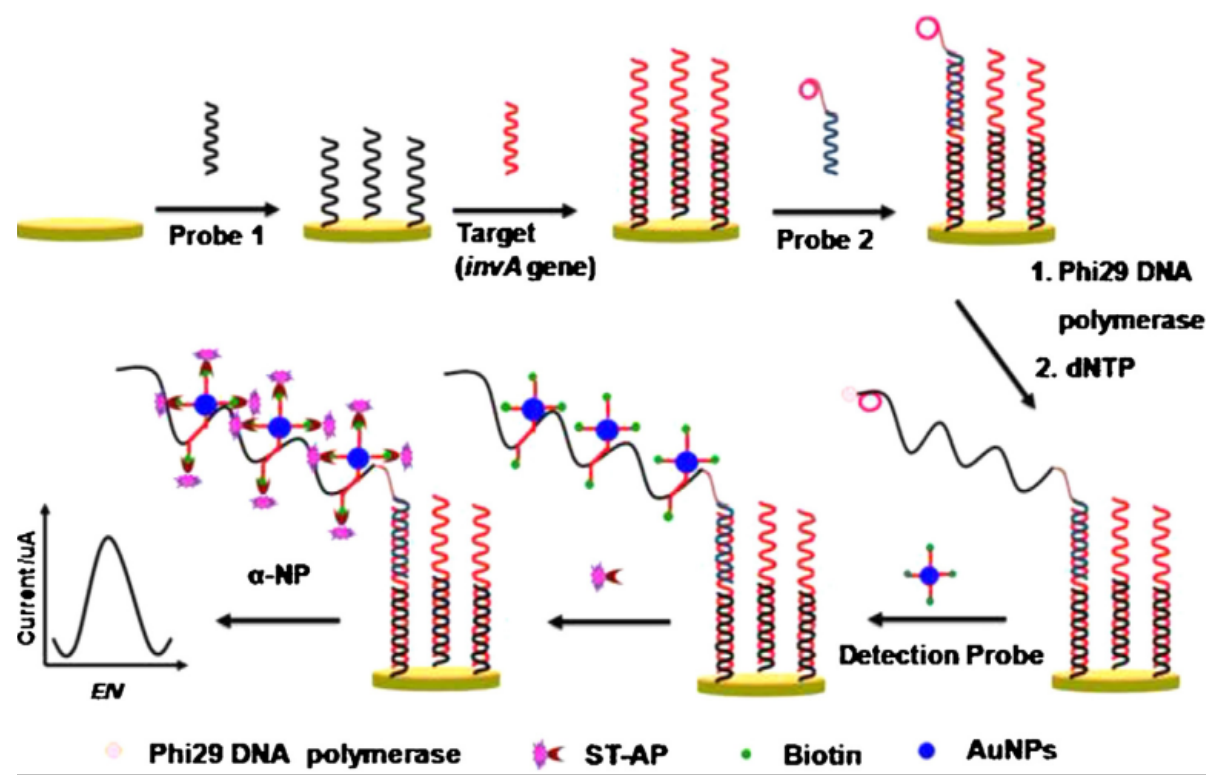

Figure 7 Scheme of the designed strategy for detection of Salmonella. ${ }^{105}$ 
Development of novel ultrasensitive EC sensors provides a great opportunity for quick and specific sensing of cancer molecular markers (CMMs), which early detection is of great importance for the success of cancer therapy. ${ }^{118}$ Johari-Ahar et al. ${ }^{119}$ modified an Au electrode with mercaptopropionic acid (MPA), and then consecutively conjugated with silica coated AuNPs (AuNPs@SiO $)$, CdSe QDs and anti-CA-125 monoclonal antibody (mAb). Successive conjugation of AuNP@ $\mathrm{SiO}_{2}, \mathrm{CdSe}$ QD and anti-CA-125mAb onto the Au electrode resulted in sensitive detection of CA-125 with aLOD of $0.0016 \mathrm{UmL}^{-1}$. Serafin et al. ${ }^{120}$ presented an immuno reagent label-free strategy to construct a dual EC immunosensor using car-bon nanotube screen printed electrodes modified with AuNPs and PEDOT NPs for the multiplexed determination of human growth hormone and prolactin. ${ }^{120}$ The ECL of luminol promoted by AuNMs was reported by Cui et al. ${ }^{121}$ The ECL intensity of luminol was found to be enhanced by $\sim 2$ to 3 orders with catalysis of AuNPs. Li et al. ${ }^{122}$ proposed a simple and sensitive sandwich-type ECL immunosensor for detection of cancer antigen 125 on a nanoporous Au modified glassy carbon electrode. ${ }^{122}$ Gao et al. ${ }^{123}$ prepared an ultrasensitive luminol ECL immunosensor by using carboxyl group functionalized MWCNTs as platform and glucose oxidase supported on AuNPs decorated MWCNTs as labels. ${ }^{123}$ The results indicated that this developed sensor exhibited sensitive and stable response for detection of $\alpha$-1-fetoprotein (AFP), ranging from 0.0001 to $80 \mathrm{ngmL}^{-1}$ with a LOD down to $0.03 \mathrm{pgmL}^{-1}$. Gui et al. ${ }^{124}$ constructed a simple and sensitive ECL biosensor for

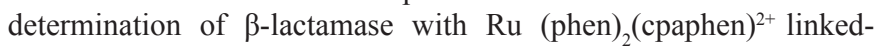
ampicillin (Ru-Amp). ${ }^{124}$ In their work, Ru-Amp complex act not only as a specific recognition element for $\beta$-lactamase but also as the ECL luminescent reagent. The biosensor displayed excellent sensitivity of a concentration variation from $50 \mathrm{pgmL}^{-1}$ to $100 \mathrm{nmL}^{-1}$ with a high sensitivity of $37 \mathrm{pgmL}^{-1}$. Beside the organic dye, noble metal NPs could greatly affect ECL behavior of semiconductor QDs. ${ }^{125}$ The ECL enhancement by LSPR of AuNPs was investigated and termed as surface enhanced ECL (SEECL). ${ }^{126}$ The LSPR of AuNPs was found to increase both the excitation rate and the emission factor of luminophores, and thus the ECL intensity was enhanced greatly. Based on this SEECL effect, a method was developed for ultrasensitive detection of $\mathrm{Hg}^{2+}$ in drinking water. ${ }^{127}$ Moreover, a SEECL sensor was prepared for detection of carcinoembryonic antigen (CEA). In this sensor, $\mathrm{Ru}(\mathrm{bpy})_{3}{ }^{2+}$-doped $\mathrm{SiO}_{2} \mathrm{NPs}\left(\mathrm{Ru} @ \mathrm{SiO}_{2}\right)$ act as ECL luminophores, while AuNPs were used as LSPR source to enhance the ECL signal. Two different types of aptamers specific to CEA were modified on the surface of $\mathrm{Ru} @ \mathrm{SiO}_{2}$ and AuNPs, respectively. A LOD of $1.52 \times 10^{-6} \mathrm{ng} / \mathrm{mL}$ of CEA in human serum was achieved. ${ }^{128}$ Electrochemical kinase analysis is of special interest in monitoring biological activity in process of life. Measurements of phosphorylation reactions based on oxidation current of electroactive species such as tyrosine, ${ }^{129}$ ferrocene, ${ }^{130}$ etc., which are conjugated on the substrate during the phosphorylation processes, have been reported. ${ }^{131}$ To simplify the detection procedure, AuNPs labeled phosphorylation process for the kinase assay was designed by measuring the redox currents of AuNPs. ${ }^{132}$ However, the sensitivity of the developed method was relatively low. In order to improve the analytical performance, a DNA-based strategy was also described by the chronocoulometric response of $\left[\mathrm{Ru}\left(\mathrm{NH}_{3}\right)_{6}\right]^{3+}$ absorbed on the DNA-AuNPs that linked with the phosphorylated peptide by $\mathrm{Zr}^{4+} .{ }^{133}$ Despite the improvement of these methods, it still remains great challenge to develop sensitive, accurate and rapid methods for the profiling of kinase activity and inhibition. Most recently, Wang et al. ${ }^{134}$ built a highly sensitive EC sensor for monitoring kinase activity based on DNA induced AuNPs polymeric network block signal amplification. ${ }^{134}$ In this strategy, the current signal of EC sensor was significantly amplified to afford a highly sensitive electrochemical analysis of kinase activity, due to its excellent electro activity and high accommodation of the DNA AuNPs polymeric network block for $\left[\mathrm{Ru}\left(\mathrm{NH}_{3}\right)_{6}\right]^{3+}$. Notably, the proposed EC sensor presented a low LOD of $0.03 \mathrm{UmL}^{-1}$ for protein kinase A (PKA) activity and excellent stability even in cell lysates and serum samples. Jing et al. ${ }^{135}$ developed a sensitive and selective electrochemical method for detection of DNA methylationas well as determination of DNA methyltransferase (MTase) activity and screening of MTase inhibitor. ${ }^{135}$ In this work, methyleneblue (MB) was employed as electrochemical indicator and DNA-modified AuNPs were used as signal amplification unit because the DNA strands in this composite had strong adsorption ability toward MB (Figure 8). The differential pulse voltammetry signal demonstrated a linear relationship with logarithm of DNA methylation concentration ranging from 0.075 to $30 \mathrm{U} / \mathrm{mL}$, achieving a LOD of $0.02 \mathrm{U} / \mathrm{mL}$ (Figure 8 ). Overall, the LSPR feature and excellent biocompatibility of AuNMs provide great chance for improving the analytical performance of EC sensors. Currently, only spherical AuNPs have been extensively applied into fabrication of EC sensor. Certainly, other shaped AuNMs possesses specific physiochemical property, and may offer additional application option in signal amplification of ECL or EC sensors.

\section{AuNMs based imaging}

Recently, AuNMs have been highlighted in biological imaging as a contrast agent due to their unique optical property. ${ }^{136}$ Biological imaging with simultaneous diagnosis and therapy will provide the multimodality needed for accurate targeted therapy. ${ }^{137}$ AuNMs have been considered as one of the best contrast agents for disease diagnosis, and functionalization of AuNMs becomes essential for application of AuNMs in computed tomography (CT), X-ray and SERS imaging. ${ }^{138}$ As one of the most reliable imaging modes, CT has been widely used owing to its high spatial and density resolution. For $\mathrm{CT}$ imaging, contrast agents are generally required to increase the density of the area to be imaged to improve the diagnostic accuracy. The commercially available CT imaging agents used in clinics are usually iodinated small molecules (e.g. omnipaque) that have severe drawbacks such as renal toxicity at a high concentration, short imaging time and non-specificity. ${ }^{139}$ For improved CT imaging, some inorganic NPs such as AuNPs ${ }^{140,141}$ have been used as contrast agents because of their higher X-ray attenuation coefficients than those of iodine-based small molecular agents. AuNPs radio sensitization represents a novel technique in enhancement of ionizing radiation dose and its effect on biological systems. ${ }^{142}$ From the first demonstration of AuNPs as a radiation contrast agent, AuNPs radio enhancement/sensitization has become an increasing area of investigation as an approach to increase the effectiveness of ionizing radiation in biological systems. ${ }^{143}$ For example, dendrimers of well-defined structure have been used as templates or stabilizers to form dendrimer-entrapped AuNPs ${ }^{144,145}$ for CT imaging applications, especially for blood pool imaging and tumor imaging. Peng et al. reported a facile approach to synthesize the dendrimer-stabilized AuNPs through use of amine-terminated fifth-generation poly (amidoamine) (PAMAM) dendrimers modified 
by diatrizoic acid as stabilizers for enhanced CT imaging. ${ }^{146} \mathrm{Li}$ et al. ${ }^{147}$ constructed Au-coated iron oxide $\left(\mathrm{Fe}_{3} \mathrm{O}_{4} @ \mathrm{Au}\right)$ nano roses to integrate five distinct functions including aptamer-based targeting, magnetic resonance imaging (MRI), optical imaging, photo thermal therapy and chemotherapy into one single probe. ${ }^{147}$ Zhang et al. ${ }^{148}$ introduced design and synthesis of branched polyethyleneimine (PEI)-stabilized AuNPs modified with polyethylene glycol (PEG) for blood pool, lymph node and tumor CT imaging. ${ }^{148}$

Despite recent advances on the different shapes of AuNPs in improving CT attenuation differences or radiotherapy damage of tumors, few studies have systematically investigated the size effect of spherical AuNPs on CT imaging and radio sensitization. ${ }^{149,150}$ In most cases, AuNPs concentration effect was examined for evaluating enhancement performances. ${ }^{151}$ Some published results indicated that great difference existed even with the same concentration of AuNPs with different size. ${ }^{152}$ In 2016, Dou et al. ${ }^{153}$ explored and confirmed the distinctive size-dependent effects based on a series of spherical AuNPs for enhanced CT imaging and radiotherapy (Figure 9). ${ }^{153}$ The result indicated that AuNPs had great size-dependent enhancement on CT imaging and radiotherapy (RT) in the size range of 3-50nm. Interestingly, AuNPs with asize of $\sim 13 \mathrm{~nm}$ could simultaneously possess superior CT contrast ability and significant radioactive disruption. AuNPs have been studied as potential contrast agents for $\mathrm{X}$-ray imaging, because they are nontoxic and have a higher atomic number and X-ray absorption coefficient compared with typical iodine-based contrast agents. ${ }^{154}$ Rand et al. ${ }^{155}$ proposed that the enhanced sensitivity of the X-ray scatter imaging technique over that of typical absorption based X-ray imaging could reduce the amount of AuNPs required for visible contrast. ${ }^{155}$ Therefore, they developed an imaging technique for the early diagnosis of hepatocellular carcinoma that utilized surface-modified AuNPs in combination with X-ray imaging. Tissues labeled with these electron-dense particles showed enhanced X-ray scattering over normal tissues, distinguishing cells containing AuNPs from cells without Au in X-ray scatter images. This approach could enable in vivo detection of tumors as small as a few millimeters in size. In 2012, Chien et al. ${ }^{156}$ used AuNPs as high-resolution X-ray imaging contrast agents for analysis of tumor-related micro-vasculature. ${ }^{156}$ AuNMs hold great interest in imaging field thanks to their characteristics such as mono dispersity, stability, minimal toxicity and excellent contrasting for transmission electron microscopy (TEM) analysis, which enable tracking with unprecedented resolution on dynamic of uptake, intracellular sorting and potential secretion..$^{157,158}$

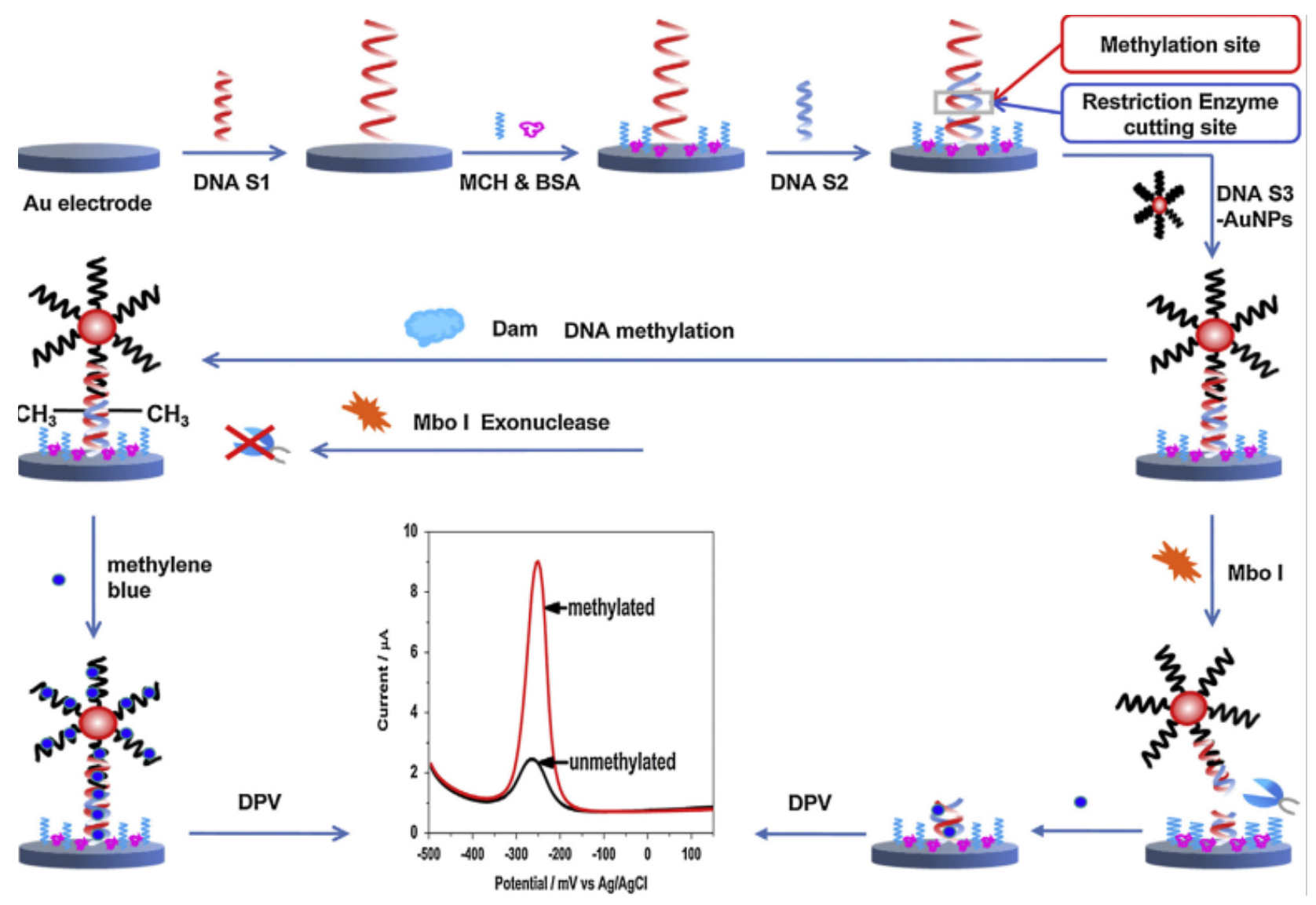

Figure 8 Scheme of the developed method for detection of DNA methylation and assay of DNA methyltransferase activity. ${ }^{135}$ 
a

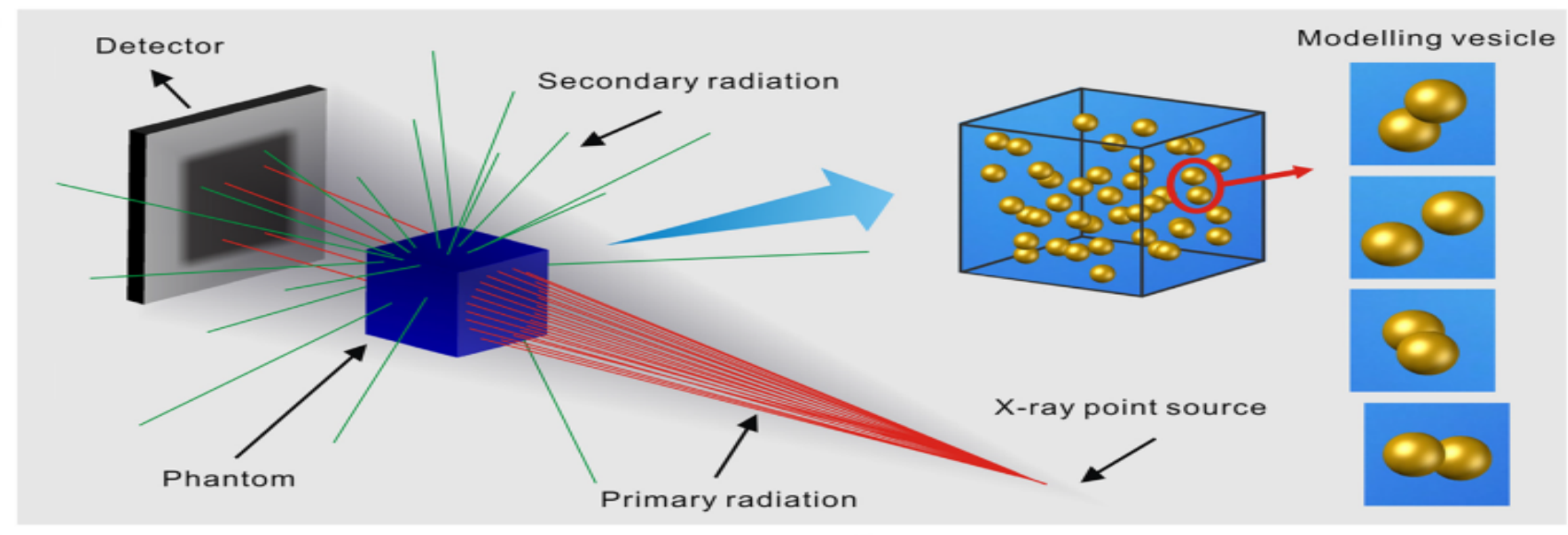

b

d
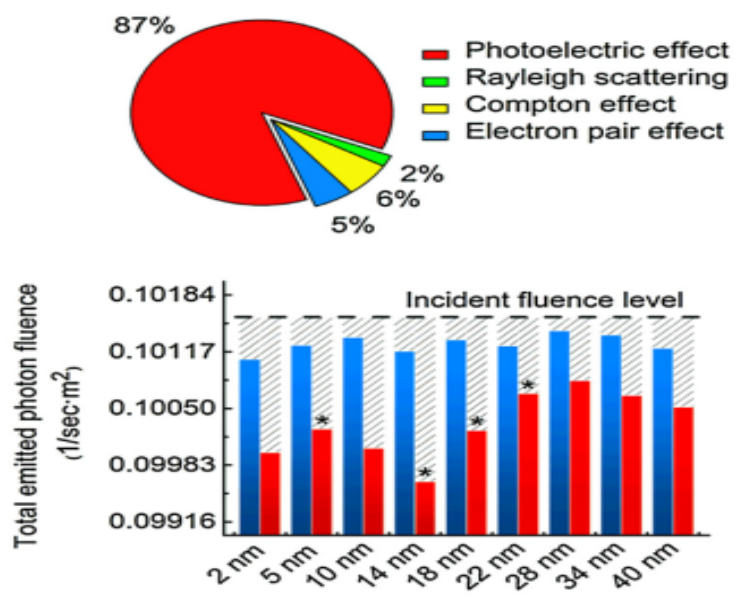

$\mathbf{f}$

h
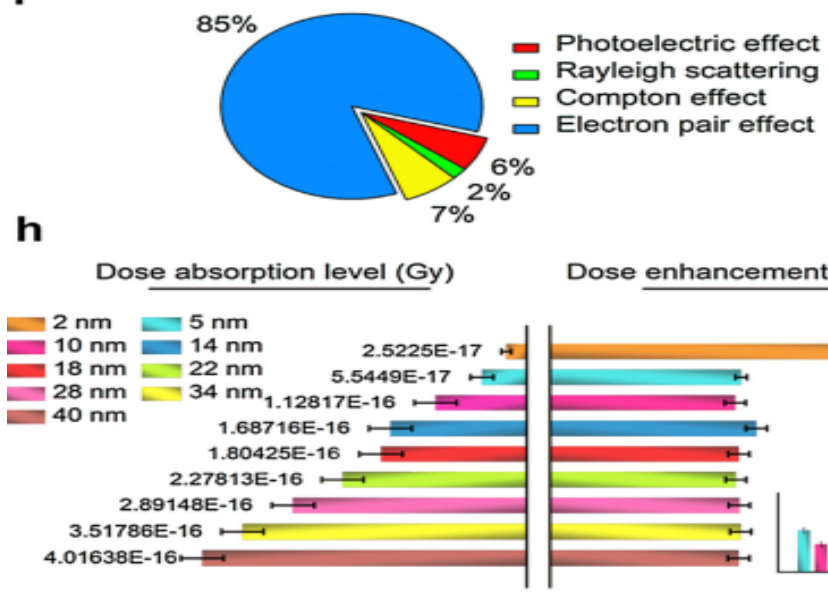

Dose enhancement ratio (DER)

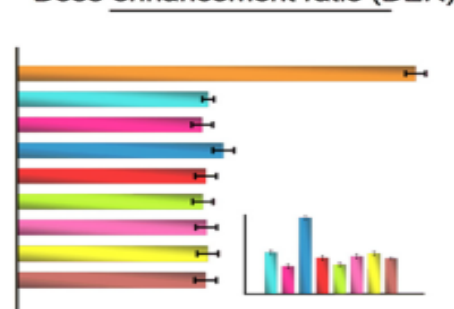

C
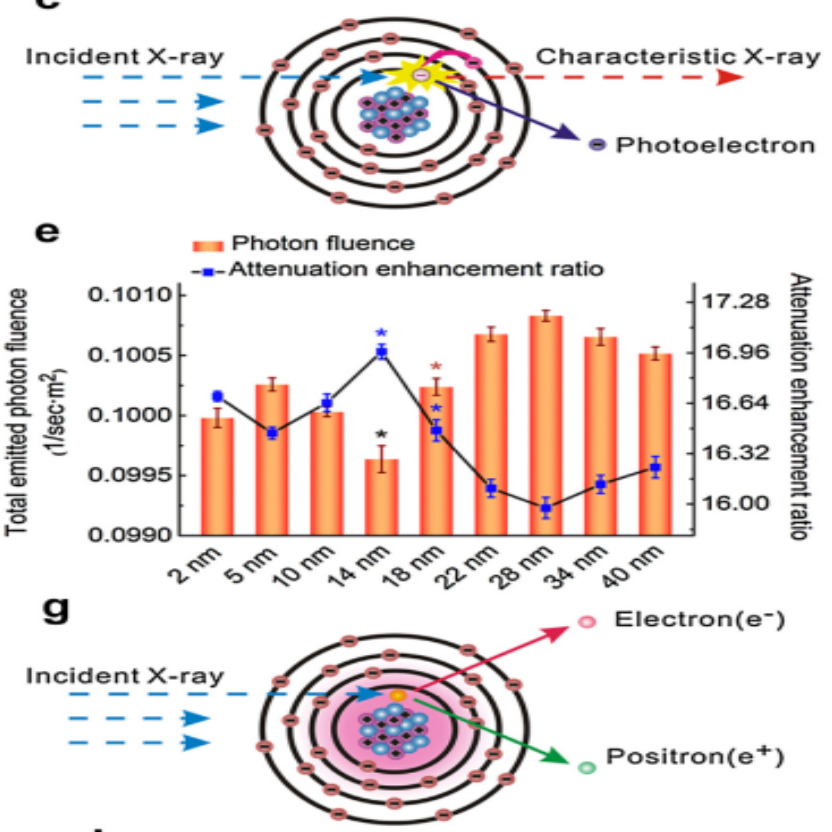

i

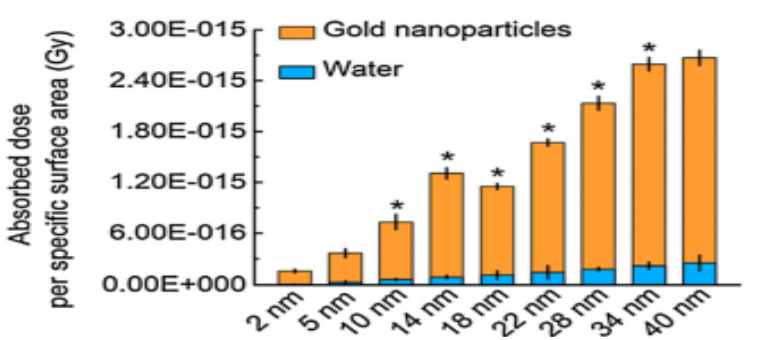

Figure 9 Monte Carlo simulation to evaluate size-dependent enhancement. (A) Scheme showing a phantom filled with a AuNP aqueous suspension that may trigger completely different secondary radiation depending on primary radiation energy irradiated from the X-ray point source for CT detection or radiotherapy.A simulated "modeling vesicle" containing two particles randomly distributed, representative of the system inhomogeneity based on particle sizes. (B) X-ray attenuation mainly coming from a photoelectric effect under the kilo voltage energy radiation for CT imaging, with little influence by other interactions including Rayleigh scattering, Compton scattering and electron pair effect. (C) Photoelectric effect generation. Inner-shell electrons receive energy from the incident $\mathrm{X}$-rays, which are subsequently ejected from the atom as a photoelectron, 
Recent results gathered on the interaction of AuNPs with different synthetic lipid membranes ${ }^{159}$ or cell lines demonstrate that not only properties such as size, charge and chemical functionality, but also the arrangement of organic ligands on NP surface, dictate the internalization route. ${ }^{160}$ Moreover, surface functionalization may influence the fate of AuNP upon cell uptake, for instance, HUVEC cells treated with AuNPs coated by different peptides display distinct exocytosis profiles. ${ }^{161}$ Recently, Marchesano et al. ${ }^{162}$ observed inward and outward trafficking of AuNPs at whole animal level using the small invertebrate Hydra vulgaris. ${ }^{162}$ AuNPs are also excellent candidates as contrast agents for SERS imaging. Yigit et al. ${ }^{163}$ synthesized conjugates of AuNMs and 3,3-diethylthiatricarbocyanine iodide (AuNM-DTTC) that were used as a bimodal contrast agent for in vivo MRI and Raman spectroscopy. ${ }^{163}$ The probe consisted of MRIactive super paramagnetic iron oxide NPs, stably complexed with AuNM-DTTC. The Au component served as a substrate for a Raman active dye molecule to generate a SERS effect. The synthesized probe produced T2 weighted contrast and was simultaneously used as a SERS active material both in silico and in vivo. Kong et al. ${ }^{164}$ prepared metal carbonyl based biotags by combining osmium carbonyl clusters and AuNPs, as an example of an organometallic-AuNPs (OMAuNPs) conjugate used to cell SERS imaging. ${ }^{164}$ It showed clearly the advantage of transition-metal carbonyl compounds, for which the $\mathrm{CO}$ stretching vibration signal was well separated from other molecular vibrational modes of the cells in live-cell imaging.

\section{Disadvantage of using AuNMs}

A big disadvantage of using AuNMs as the optical contrast agents is their high photo thermal conversion efficiency under resonance excitation, which may perturb or even damage the biological species being imaged. ${ }^{165}$ Thus, to minimize unwanted heat-ing in bio imaging applications, laser irradiation time is typically set to be in the range of $0.1-10 \mathrm{~S}$, which either lowers the SERS signal contrast or is simply not suited for image-guided tumor resection in intraoperative settings, considering the longer timescales $(1-2 h)$ associated with such procedures. ${ }^{166,167}$ While resonant nanostructures are certainly desirable for the ranostic applications, having the ability to tune the photo thermal effect of SERS probes while preserving their high SERS activity is critical to avoid unwanted heating during imaging. Oh et al. ${ }^{168}$ designed and synthesized plasmonic AuNPs with ultra small (typically, $\sim 1 \mathrm{~nm}$ ) interior gap using thiolated DNA and obtained the relationships between these noble metal nano gap structures and plasmonic signals from these structures. ${ }^{168}$ In 2015, Tian et al. ${ }^{169}$ demonstrate bio enabled synthesis of a novel class of ultra-bright SERS probes with built-in and accessible electromagnetic hotspots formed by densely packed satellite NPs grown on a plasmonic core. ${ }^{169}$ Through the rational choice of the shape of the core, the LSPR wavelength of Au superstructures was tuned to be either off or on-resonant with the NIR excitation without sacrificing their high SERS activity. Consequently, the photo thermal efficiency of these ultra-bright SERS tags could be tuned to realize either contrast agents with minimal heating and perturbation, or multifunctional the ranostic agents that imaged and photo thermally killed the targeted cells.

\section{Conclusions}

I. The outstanding characteristics of AuNMs make them promising candidates as the signal reporters, enhancement materials or others involved with bioassay, food safety, environmental monitoring and medical science.

II. 2-a series of novel and sophisticated synthesis methods for AuNMs have been developed, but how to precisely control the mono dispersed size and morphology and to achieve high quality of AuNMs with vivid color (LSPR) is still a crucial but challengeable issue for their application in analytical science. The AuNMs with precisely-controlled vivid color possess abundant optical information and can encode biological or chemical recognition units to develop robust analytical methods in food safety, clinic diagnosis and so on. In addition, we are far from controllable assembly of AuNMs into the desirable structures of the collective properties. ${ }^{170}$

III. AuNMs have shown excellent performance in enhancement of SERS signal to improve detection sensitivity. Therefore, AuNMs in combination with other functional metal or organic nanomaterials, such as $\mathrm{Si}, \mathrm{Al}, \mathrm{MOF}$ and so on, to develop multimodal composite nanomaterials become a new trend in high sensitive SERS detection of various target analytes, ${ }^{171,172}$ as well as in high resolution bio imaging in the future.

IV. AuNMs offer a rapid, efficient, cost-effective and robust sensing platform for detection of different chemicals and bio-markers due to their unique chemical, physical and optical properties. It is noted that the sensitive, stable and multiplex assay of target analytes is highly desirable. To meet these requirements, new multifunctional AuNMs must be developed and used as LFICA label, colorimetric sensing readout and signal amplification of EC sensor, etc.

V. AuNCs as excellent fluorescent probe materials have shown great potential in analytical science. ${ }^{173}$ However, extensive efforts must be made for developing novel synthesis methods to achieve high quality AuNCs with remarkable QY and extraordinary stability. In addition, the coordination nature of the AuNCs-protection group complexes implies that the stability of metal NCs is affected by the presence of other strong competing ligands in aqueous solution. The intracellular stability of AuNCs hence becomes a serious issue. With development of technologies of synthesis and surface modification, AuNCs will be widely employed as alternatives to conventional fluorophores in analytical science including biosensors, bio imaging and so on.

VI. We notice that though many new analytical methods based on AuNMs have been developed in the laboratories, such materials are not used as much in an industrial setting. There must be more scope for these platforms to improve, for example, cost of preparing the AuNMs based sensors, reproducibility of different batches for sensor production, and stability of long-term storage of the AuNMs based sensors, and so on.

VII. The mechanism of DNA-guided AuNMs synthesis not only provide deep understanding of the interactions between the DNA and nanomaterials but also allow better control of the shapes and surface properties of many nanomaterials. 8- AuNMs with excellent features have prompted great development of analytical sciences and have stood at a critical juncture, with a 
vast amount of researches that form a solid foundation for future work. Great deals of efforts need to be paid to push on to take AuNMs-based analytical technologies from lab to market.

\section{Acknowledgments}

None.

\section{Conflict of Interest}

The authors declared there is no conflict of interest.

\section{References}

1. Madkour LH. Vision for life sciences: interfaces between nanoelectronic and biological systems. Glob Drugs Therap. 2017;2(4):1-4.

2. Sardar R, Funston AM, Mulvaney P, et al. Gold nanoparticles: past, present, and future. Langmuir. 2009;25(24):13840-13851.

3. Wang P, Sun X, Su X, et al. Advancements of molecularly imprinted polymers in the food safety field. Analysts. 2016;141:3540-3553.

4. Zhang L, Wang E. Metal nanoclusters: New fluorescent probes for sensors and bioimaging. Nano Today. 2014;9(1):132-157.

5. Ballou S, Goodpaster J, MacCrehan W, et al. Forensic analysis. Anal Bioanal Chem. 2003;376(8):1149-1150.

6. Burnworth M, Rowan SJ, Weder C. Fluorescent sensors for the detection of chemical warfare agents. Chemistry. 2007;13(28):7828-7836.

7. Zhang Z, Liu J, Feng T, et al. Time-Resolved Fluoroimmunoassay as an Advantageous Analytical Method for Assessing the Total Concentration and Environmental Risk of Fluoroquinolones in Surface Waters. Environ Sci Technol. 2013;47(1):454-462.

8. Anker JN, Hall WP, Lyandres O, et al. Biosensing with plasmonic nanosensors. Nat Mater. 2008;7(6):442-453.

9. Debouttiere PJ, Roux S, Vocanson F, et al. Design of Gold Nanoparticles for Magnetic Resonance Imaging. Adv Funct Mater. 2006;16(18):2330 2339 .

10. Qian X, Li J, Nie S. Stimuli-responsive SERS nanoparticles: conformational control of plasmonic coupling and surface Raman enhancement. J Am Chem Soc. 2009;131(22):7540-7541.

11. Thomas KG, Kamat PV. Making Gold Nanoparticles Glow: Enhanced Emission from a Surface-Bound Fluoroprobe. $J$ Am Chem Soc. 2000;122(11):2655-2656.

12. Ipe BI, Yoosaf K, Thomas KG. Functionalized gold nanoparticles as phosphorescent nanomaterials and sensors. $J$ Am Chem Soc. 2006;128(6):1907-1913.

13. Kisailus D, Najarian M, Weaver JC, et al. Functionalized Gold Nanoparticles Mimic Catalytic Activity of a Polysiloxane-Synthesizing Enzyme. Adv Mater. 2005;17(10):1234-1239.

14. Qian X, Peng XH, Ansari DO, et al. In vivo tumor targeting and spectroscopic detection with surface-enhanced Raman nanoparticle tags. Nat Biotechnol. 2008;26(1):83-90.

15. Haghighi B, Bozorgzadeh S. Enhanced electrochemiluminescence from luminol at multi-walled carbon nanotubes decorated with palladium nanoparticles: A novel route for the fabrication of an oxygen sensor and a glucose biosensor. Anal Chim Acta. 2011;697(1-2):90-97.
16. Tang J, Tang DP, Su BL, et al. Enzyme-free electrochemical immunoassay with catalytic reduction of p-nitrophenol and recycling of p-aminophenol using gold nanoparticles-coated carbon nanotubes as nanocatalysts. Biosens Bioelectron. 2011;26(7):3219-3226.

17. Zhu Y, Chandra P, Song KM, et al. Label-free detection of kanamycin based on the aptamer-functionalized conducting polymer/gold nanocomposite. Biosens Bioelectron. 2012;36(1):29-34.

18. Sun $\mathrm{X}$, Li F, Shen G, et al. Aptasensor based on the synergistic contributions of chitosan-gold nanoparticles, graphene-gold nanoparticles and multi-walled carbon nanotubescobalt phthalocyanine nanocomposites for kanamycin detection. Analyst. 2014;139(1):299 308 .

19. Roushani M, Shahdostfard F. A highly selective and sensitive cocaine aptasensor based on covalent attachment of the aptamer-functionalized AuNPs onto nanocomposite as the support platform. Anal Chim Acta. 2015;853:214-221.

20. Wang Y, Knoll W, Dostalek J. Bacterial pathogen surface plasmonresonance biosensor advanced by long range surface plasmons and magneticnanoparticle assays. Anal Chem. 2012;84(19):8345-8350.

21. Yang X, Wang Q, Wang K, et al. Enhanced surface plasmon resonance with the modified catalytic growth of $\mathrm{Au}$ nanoparticles. Biosens Bioelectron. 2007;22(6):1106-1110.

22. Jung J, Na K, Lee J, et al. Enhanced surface plasmon resonance by $\mathrm{Au}$ nanoparticles immobilized on a dielectric $\mathrm{SiO}_{2}$ layer on a gold surface. Anal Chim Acta. 2009;651(1):91-97.

23. Shen L, Chen Z, Li YH, et al. Electrochemical DNAzyme sensor for lead based on amplification of DNA-Au bio-bar codes. Anal Chem. 2008;80(16):6323-6328.

24. Tang S, Tong P, Lu W, et al. A novel label-free electrochemical sensor for $\mathrm{Hg}^{2+}$ based on the catalytic formation of metal nanoparticle. Biosens Bioelectron. 2014;59:1-5.

25. Wang N, Lin M, Dai H, et al. Functionalized gold nanoparticles/reduced graphene oxide nanocomposites for ultrasensitive electrochemical sensing of mercury ions based on thymine-mercury-thymine structure. Biosens Bioelectron. 2016;79:320-326.

26. Wang S, Wang Y, Zhou L, et al. Fabrication of an effective electrochemical platform based on graphene and AuNPs for high sensitive detection of trace $\mathrm{Cu}^{2+}$. Electrochim Acta. 2014;132:7-14

27. Yang Y, Zhang S, Kang M, et al. Selective detection of silver ions using mushroom-like polyaniline and gold nanoparticle nanocomposite-based electrochemical DNA sensor. Anal Biochem. 2015;490:7-13.

28. Kimling J, Maier M, Okenve B, et al. Turkevich method for gold nanoparticle synthesis revisited. J Phys Chem B. 2006;110(32):1570015707.

29. Kumar S, Gandhi KS, Kumar R. Modeling of formation of gold nanoparticles by citrate method. Ind Eng Chem Res. 2007;46(10):31283136.

30. Schmid G. Large clusters and colloids. Metals in the embryonic state. Chem Rev. 1992;92(8):1709-1727.

31. Schmid G, Chi LF. Metal clusters and colloids. Adv Mater. 1998;10(7):515-526.

32. Brust M, Fink J, Bethell D, et al. Synthesis and reactions of functionalised gold nanoparticles. J Chem Soc Chem Commun. 1995;16:1655-1656. 
33. Saha K, Agasti SS, Kim C, et al. Gold nanoparticles in chemical and biological sensing. Chem Rev. 2012;112(5):2739-2779.

34. Daniel MC, Astruc D. Gold nanoparticles: assembly, supramolecular chemistry, quantum-size-related properties, and applications toward biology, catalysis, and nanotechnology. Chem Rev. 2004;104(1):293346.

35. Burda C, Chen X, Narayan R, et al. Chemistry and properties of nanocrystals of different shapes. Chem Rev. 2005;105(4):1025-1102.

36. Skrabalak SE, Chen J, Au L, et al. Gold Nanocages for Biomedical Applications. Adv Mater. 2007;19(20):3177-3184.

37. Sun Y, Xia Y. Shape-controlled synthesis of gold and silver nanoparticles. Science. 2002;298(5601):2176-2179.

38. Malikova N, Pastoriza-Santos I, Schierhorn M, et al. Layer-by-layer assembled mixed spherical and planar gold nanoparticles: control of interparticle interactions. Langmuir. 2002;18(9):3694-3697.

39. Manna L, Milliron D, Meisel A, et al. Controlled growth of tetrapodbranched inorganic nanocrystals. Nat Mater. 2003;2(6):382-385.

40. Cao YW, Jin R, Mirkin CA. DNA-modified core-shell Ag/Au nanoparticles. J Am Chem Soc. 2001;123(32):7961-7962.

41. Zhang L, Niu W, Xu G. Synthesis and applications of noble meta nanocrystals with high-energy facets. Nano Today. 2012;7(6):586-605.

42. Lai J, Niu W, Luque R, et al. Solvothermal synthesis of metal nanocrystals and their applications. Nano Today. 2015;10(2):240-267.

43. Kim F, Song JH, Yang P. Photochemical synthesis of gold nanorods. $J$ Am Chem Soc. 2002;124(48):14316-14317.

44. Niu W, Zheng S, Wang D, eta 1. Selective synthesis of single-crystalline rhombic dodecahedral, octahedral, and cubic gold nanocrystals. $J$ Am Chem Soc. 2009;131(2):697-703.

45. Niu W, Chua YAA, Zhang W, et al. Highly Symmetric Gold Nanostars Crystallographic Control and Surface-Enhanced Raman Scattering Property. J Am Chem Soc. 2015;137(33):10460-10463.

46. Zheng J, Petty JT, Dickson RM. High quantum yield blue emission from water-soluble Au8 nanodots. J Am Chem Soc. 2003;125(26):7780-7781.

47. Xie J, Zheng Y, Ying JY. Protein-directed synthesis of highly fluorescent gold nanoclusters. J Am Chem Soc. 2009;131(3):888-889.

48. Varnavski O, Ramakrishna G, Kim J, et al. Critical Size for the Observation of Quantum Confinement in Optically Excited Gold Clusters. J Am Chem Soc. 2010;132(1):16-17.

49. Tang Z, Xu B, Wu B, et al. Synthesis and Structural Determination of Multidentate 2,3-Dithiol-Stabilized Au Clusters. J Am Chem Soc. 2010;132(10):3367-3374.

50. Yu Y, Luo Z, Chevrier DM, et al. Identification of a highly luminescent Au22(SG)18 nanocluster. J Am Chem Soc. 2014;136(49):1246-1249.

51. Pyo K, Thanthirige VD, Kwak K, et al. Ultrabright Luminescence from Gold Nanoclusters: Rigidifying the Au(I)-Thiolate Shell. J Am Chem Soc. 2015;137(25):8244-8250.

52. Tan LH, Xing H, Lu Y. DNA as a Powerful Tool for Morphology Control, Spatial Positioning, and Dynamic Assembly of Nanoparticles. Acc Chem Res. 2014;47(6):1881-1890.

53. Wang Z, Tang L, TanLH, et al. Discovery of the DNA "Genetic Code" for Abiological Gold Nanoparticle Morphologies. Angew Chem Int Ed. 2012;51(36):9078-9082.
54. Song T, Tang L, Tan LH, et al. DNA-Encoded Tuning of Geometric and Plasmonic Properties of Nanoparticles Growing from Gold Nanorod Seeds. Angew Chem Int Ed. 2015;54(28):8114-8118.

55. Tan L, Yue Y, Satyavolu NSR, et al. Mechanistic Insight into DNAGuided Control of Nanoparticle Morphologies. J Am Chem Soc. 2015;137(45):14456-14464.

56. Ye $\mathrm{X}$, Jin L, Cagalayan $\mathrm{H}$, et al. Improved size-tunable synthesis of monodisperse gold nanorods through the use of aromatic additives. ACS Nano. 2012;6(3):2804-2817.

57. Krishna KS, Navin CV, Biswas S, et al. Millifluidics for time-resolved mapping of the growth of gold nanostructures. J Am Chem Soc. 2013;135(14):5450-5456.

58. Johnston CW, Wyatt MA, Li X, et al. Gold biomineralization by a metallophore from a gold-associated microbe. Nat Chem Biol. 2013;9(4):241-243.

59. Zhang MX, Cui R, Tian ZQ, et al. Kinetics-Controlled Formation of Gold Clusters Using a Quasi-Biological System. Adv Funct Mater. 2010;20(21):3673-3677.

60. Shin Y, Lee C, Yang MS, et al. Two-dimensional hyper-branched gold nanoparticles synthesized on a two-dimensional oil/water interface. Sci Rep. 2014;4:6119.

61. Abdelaziz R, Disci-Zayed D, Hedayati MK, et al. Green chemistry and nanofabrication in a levitated Leidenfrost drop. Nat Commun $2013 ; 4: 2400$.

62. Kim JH, Twaddle KM, Hu J, et al. Sunlight-induced synthesis of various gold nanoparticles and their heterogeneous catalytic properties on a paper-based substrate. ACS Appl Mater Interfaces. 2014;6(14):11514 11522.

63. Gao C, Zhang Q, Lu Z, et al. Templated synthesis of metal nanorods in silica nanotubes. J Am Chem Soc. 2011;133(49):19706-19709.

64. Liu J, Qiao SZ, Hartono SB, et al. Monodisperse yolk-shell nanoparticles with a hierarchical porous structure for delivery vehicles and nanoreactors. Angew Chem Int Ed. 2010;49(29):4981-4985.

65. Christesen SD. Raman Cross Sections of Chemical Agents and Simulants. Appl Spectrosc. 1988;42(2):318-321.

66. Yan $\mathrm{J}, \mathrm{Su} \mathrm{S}, \mathrm{He} \mathrm{S}$, et al. Nano rolling-circle amplification for enhanced SERS hot spots in protein microarray analysis. Anal Chem. 2012;84(21):9139-9145.

67. Li M, Cushing SK, Zhang J, et al. Three-dimensional hierarchical plasmonic nano-architecture enhanced surface-enhanced Raman scattering immunosensor for cancer biomarker detection in blood plasma. ACS Nano. 2013;7(6):4967-4976.

68. Luo Z, Li W, Lu D, et al. A SERS-based immunoassay for porcine circovirus type 2 using multi-branched gold nanoparticles. Microchim Acta. 2013;180(15-16):1501-1507.

69. Tang J, Sun J, Lui R, et al. New Surface-Enhanced Raman Sensing Chip Designed for On-Site Detection of Active Ricin in Complex Matrices Based on Specific Depurination. ACS Appl Mater Interfaces. 2016;8(3):2449-2455

70. Qian X, Peng XH, Ansari DO, et al. In vivo tumor targeting and spectroscopic detection with surface-enhanced Raman nanoparticle tags. Nat Biotechnol. 2008;26(1):83-90.

71. Lim DK, Jeon KS, Kim HM, et al. Nanogap-engineerable Raman-active nanodumbbells for single-molecule detection. Nat Mater. 2010;9(1):60 67. 
72. Wang Y, Zhang CH, Tang LJ, et al. Enzymatic control of plasmonic coupling and surface enhanced Raman scattering transduction for sensitive detection of DNA demethylation. Anal Chem. 2012;84(20):8602-8606.

73. Seydack M. Nanoparticle labels in immunosensing using optical detection methods. Biosens Bioelectron. 2005;20(12):2454-2469.

74. Chen K, Han H, Luo Z, et al. A practicable detection system for genetically modified rice by SERS-barcoded nanosensors. Biosens Bioelectron. 2012;34(1):118-124.

75. Ye $\mathrm{S}, \mathrm{Wu} \mathrm{Y}$, Zhai $\mathrm{X}$, et al. Asymmetric signal amplification for simultaneous SERS detection of multiple cancer markers with significantly different levels. Anal Chem. 2015;87(16):8242-8249.

76. Wen CY, Wu LL, Zhang ZL, et al. Quick-response magnetic nanospheres for rapid, efficient capture and sensitive detection of circulating tumor cells. ACS Nano. 2014;8(1):941-949.

77. Wang X, Qian X, Beitler JJ, et al. Detection of circulating tumor cells in human peripheral blood using surface-enhanced Raman scattering nanoparticles. Cancer Res. 2011;71(5):1526-1532.

78. Wu X, Luo L, Yang S, et al. Improved SERS Nanoparticles for Direct Detection of Circulating Tumor Cells in the Blood. ACS Appl Mater Interfaces. 2015;7(18):9965-9971.

79. Dudak FC, Boyaci IH. Rapid and label-free bacteria detection by surface plasmon resonance (SPR) biosensors. Biotechnol J. 2009;4(7):10031011.

80. Ashley J, Li SF. An aptamer based surface plasmon resonance biosensor for the detection of bovine catalase in milk. Biosens Bioelectron. $2013 ; 48: 126-131$.

81. McPhillips J, Murphy A, Jonsson MP, et al. High- performance biosensing using arrays of plasmonic nanotubes. ACS Nano. 2010;4(4):2210-2216.

82. Sim HR, Wark AW, Lee HJ. Attomolar detection of protein biomarkers using biofunctionalized gold nanorods with surface plasmon resonance. Analyst. 2010;135(10):2528-2532.

83. Shankaran DR, Gobi KVA, Miura N. Recent advancements in surface plasmon resonance immunosensors for detection of small molecules of biomedical, food and environmental interest. Sens Actuators B Chem. 2007;121(1):158-177.

84. Fernandez F, Sanchez-Baeza F, Marco MP. Nanogold probe enhanced Surface Plasmon Resonance immunosensor for improved detection of antibiotic residues. Biosens Bioelectron. 2012;34(1):151-158.

85. Jung J, Na K, Lee J, et al. Enhanced surface plasmon resonance by Au nanoparticles immobilized on a dielectric $\mathrm{SiO}_{2}$ layer on a gold surface. Anal Chim Acta. 2009;651(1):91-97.

86. Ko S, Park TJ, Kim HS, et al. Directed self-assembly of gold binding polypeptide-protein A fusion proteins for development of gold nanoparticle-based SPR immunosensors. Biosens Bioelectron. 2009;24(8):2592-2597.

87. Wang J, Munir A, Li Z, et al. Aptamer-Au NPs conjugates-enhanced SPR sensing for the ultrasensitive sandwich immunoassay. Biosens Bioelectron. 2009;25(1):124-129.

88. Liu X, Li RZ, Li L, et al. Immunoanalysis of E. coli O157: H7 Based on Au Nanoparticles Labelling Antibody Using SPR Biosensor. Chem J Chin Univ. 2013;34(6):1333-1338.

89. Law WC, Yong KT, Baev A, et al. Sensitivity improved surface plasmon resonance biosensor for cancer biomarker detection based on plasmonic enhancement. ACS Nano. 2011;5(6):4858-4864.
90. Xiang Y, Zhu X, Huang Q, et al. Real-time monitoring of mycobacterium genomic DNA with target-primed rolling circle amplification by a $\mathrm{Au}$ nanoparticle-embedded SPR biosensor. Biosens Bioelectron. 2015;66:512-519.

91. Li X, Cai W, An J, et al. Large-area synthesis of high-quality and uniform graphene films on copper foils. Science. 2009;324(5932):1312-1314.

92. Shang J, Ma L, Li J, et al. The origin of fluorescence from graphene oxide. Sci Rep. 2012;2:792.

93. Zhang J, Sun Y, Wu Q, et al. A protein A modified Au-graphene oxide composite as an enhanced sensing platform for SPR-based immunoassay. Analyst. 2013;138(23):7175-7181.

94. Xie C, Li H, Li S, et al. Surface Molecular Self-Assembly for Organophosphate Pesticide Imprinting in Electropolymerized Poly(paminothiophenol) Membranes on a Gold Nanoparticle Modified Glassy Carbon Electrode. Anal Chem. 2010;82(1):241-249.

95. Yu D, Zeng Y, Qi Y, et al. A novel electrochemical sensor for determination of dopamine based on AuNPs@SiO, core-shell imprinted composite. Biosens Bioelectron. 2012;38(1):270-277.

96. Xue C, Han Q, Wang Y, et al. Amperometric detection of dopamine in human serum by electrochemical sensor based on gold nanoparticles doped molecularly imprinted polymers. Biosens Bioelectron. 2013;49:199-203.

97. Sun X, Zhang L, Zhang H, et al. Development and application of 3-chloro-1,2-propandiol electrochemical sensor based on a polyaminothiophenol modified molecularly imprinted film. J Agric Food Chem. 2014;62(20):4552-4557.

98. Shen L, Chen Z, Li Y, et al. Electrochemical DNAzyme sensor for lead based on amplification of DNA-Au bio-bar codes. Anal Chem. 2008;80(16):6323-6328.

99. Tang S, Tong P, Lu W, et al. A novel label-free electrochemical sensor for $\mathrm{Hg} 2+$ based on the catalytic formation of metal nanoparticle. Biosens Bioelectron. 2014;59:1-5.

100. Wang N, Lin M, Dai H, et al. Functionalized gold nanoparticles/reduced graphene oxide nanocomposites for ultrasensitive electrochemical sensing of mercury ions based on thymine-mercury-thymine structure. Biosens Bioelectron. 2016;79:320-326.

101. Wang S, Wang Y, Zhou L, et al. Fabrication of an effective electrochemical platform based on graphene and AuNPs for high sensitive detection of trace $\mathrm{Cu}^{2+}$. Electrochimica Acta. 2014;132:7-14.

102. Yang Y, Zhang S, Kang M, et al. Selective detection of silver ions using mushroom-like polyaniline and gold nanoparticle nanocomposite-based electrochemical DNA sensor. Anal Biochem. 2015;490:7-13.

103. He X, Su J, Wang Y, et al. A sensitive signal-on electrochemical assay for MTase activity using AuNPs amplification. Biosens Bioelectron. 2011;28(1):298-303.

104. Wang Q, Gao F, Ni J, et al. Facile construction of a highly sensitive DNA biosensor by in-situ assembly of electro-active tags on hairpinstructured probe fragment. Sci Rep. 2016;6:22441.

105. Zhu D, Yan Y, Lei P, et al. A novel electrochemical sensing strategy for rapid and ultrasensitive detection of Salmonella by rolling circle amplification and DNA-AuNPs probe. Anal Chim Acta. 2014;846:4450 .

106. Song W, Li DW, Li YT, et al. Disposable biosensor based on graphene oxide conjugated with tyrosinase assembled gold nanoparticles. Biosens Bioelectron. 2011;26(7):3181-3186. 
107. Li C, Wei L, Liu X, et al. Simple electrochemical sensing of attomolar proteins using fabricated complexes with enhanced surface binding avidity. Chem Sci. 2015;6(7):4311-4317.

108. Myung S, Park J, Lee H, et al. Ambipolar memory devices based on reduced graphene oxide and nanoparticles. Adv Mater. 2010;22(18):2045-2049.

109. Huang KJ, Niu DJ, Liu X, et al. Direct electrochemistry of catalase at amine-functionalized graphene/gold nanoparticles composite film for hydrogen peroxide sensor. Electrochim Acta. 2011;56(7):2947-2953.

110. Cui P, Seo S, Lee J, et al. Nonvolatile memory device using gold nanoparticles covalently bound to reduced graphene oxide. ACS Nano. 2011;5(9):6826-6833

111. Zheng J, He Y, Sheng Q, et al. DNA as a linker for biocatalytic deposition of Au nanoparticles on graphene and its application in glucose detection. J Mater Chem. 2011;21:12873-12879.

112. Liu J, Fu S, Yuan B, et al. Toward a universal "adhesive nanosheet" for the assembly of multiple nanoparticles based on a proteininduced reduction/decoration of graphene oxide. $\mathrm{J}$ Am Chem Soc. 2010;132(21):7279-7281

113. Liu G, Qi M, Zhang Y, et al. Nanocomposites of gold nanoparticles and graphene oxide towards an stable label-free electrochemical immunosensor for detection of cardiac marker troponin-I. Anal Chim Acta. 2016;909:1-8

114. Karaboga MNS, Simsek CS, Sezgintürk MK. AuNPs modified, disposable, ITO based biosensor: Early diagnosis of heat shock protein 70. Biosens Bioelectron. 2016;84:22-29.

115. Wang L, Hua E, Liang M, et al. Graphene sheets, polyaniline and AuNPs based DNA sensor for electrochemical determination of BCR/ ABL fusion gene with functional hairpin probe. Biosens Bioelectron. 2014;51:201-207.

116. Su S, Zou M, Zhao H, et al. Shape-controlled gold nanoparticles supported on $\mathrm{MoS}_{2}$ nanosheets: synergistic effect of thionine and MoS and their application for electrochemical label-free immunosensing. Nanoscale. 2015;7(45):19129-19135.

117. Su S, Sun H, Cao W, et al. Dual-Target Electrochemical Biosensing Based on DNA Structural Switching on Gold Nanoparticle-Decorated $\mathrm{MoS}_{2}$ Nanosheets. ACS Appl Mater Interfaces. 2016;8(11):6826-6833.

118. Das J, Kelley SO. Protein Detection Using Arrayed Microsensor Chips Tuning Sensor Footprint to Achieve Ultrasensitive Readout of CA-125 in Serum and Whole Blood. Anal Chem. 2011;83(4):1167-1172.

119. Johari-Ahar M, Rashidi MR, Barar J, et al. An ultra-sensitive impedimetric immunosensor for detection of the serum oncomarker CA-125 in ovarian cancer patients. Nanoscale. 2015;7(8):3768-3779.

120. Serafin V, Martinez-Garcia G, Agui L, et al. Multiplexed determination of human growth hormone and prolactin at a label free electrochemical immunosensor using dual carbon nanotube-screen printed electrodes modified with gold and PEDOT nanoparticles. Analyst. 2014;139(18):4556-4563.

121. Cui H, Xu Y, Zhang ZF. Multichannel electrochemiluminescence of luminol in neutral and alkaline aqueous solutions on a gold nanoparticle self-assembled electrode. Anal Chem. 2004;76(14):4002-4010.

122. Li M, Zhang M, Ge S, et al. Ultrasensitive electrochemiluminescence immunosensor based on nanoporous gold electrode and Ru-AuNPs/ graphene as signal labels. Sens Actuators B. 2013;181:50-56.

123. Cao Y, Yuan R, Chai Y, et al. Ultrasensitive luminol electrochemiluminescence for protein detection based on in situ generated hydrogen peroxide as coreactant with glucose oxidase anchored AuNPs@MWCNTs labeling. Biosens Bioelectron. 2012;31(1):305-309.

124. Gui GF, Zhuo Y, Chai YQ, et al. A novel ECL biosensor for $\beta$-lactamase detection: Using RU(II) linked-ampicillin complex as the recognition element. Biosens Bioelectron. 2015;70:221-225.

125. Wang J, Shan Y, Zhao WW, et al. Gold Nanoparticle Enhanced Electrochemiluminescence of CdS Thin Films for Ultrasensitive Thrombin Detection. Anal Chem. 2011;83(11):4004-4011.

126. Wang D, Guo L, Huang R, et al. Surface enhanced electrochemiluminescence of Ru(bpy)3(2+). Sci Rep. 2015;5:7954.

127. Wang D, Guo L, Huang R, et al. Surface Enhanced Electrochemiluminescence for Ultrasensitive Detection of $\mathrm{Hg}^{2+}$. Electrochim Acta. 2014;150:123-128.

128. Wang D, Li Y, Lin Z, et al. Surface-Enhanced Electrochemiluminescence of Ru@SiO for Ultrasensitive Detection of Carcinoembryonic Antigen. Anal Chem. 2015;87(12):5966-5972.

129. Kerman K, Vestergaard M, Tamiya E. Label-free electrical sensing of small-molecule inhibition on tyrosine phosphorylation. Anal Chem. 2007;79(17):6881-6885.

130. Wilkins MH, Stokes AR, Wilson HR. Molecular structure of deoxypentose nucleic acids. Nature. 1953;171(4356):738-740.

131. Sessler JL, Lawrence CM, Jayawickramarajah J. Molecular recognition via base-pairing. Chem Soc Rev. 2007;36(2):314-325.

132. Kerman K, Chikae M, Yamamura S, et al. Gold nanoparticle-based electrochemical detection of protein phosphorylation. Anal Chim Acta. 2007;588(1):26-33

133. Xu X, Nie Z, Chen J, et al. A DNA-based electrochemical strategy for label-free monitoring the activity and inhibition of protein kinase. Chem Commun. 2009;45:6946-6948.

134. Wang Z, Sun N, He Y, et al. DNA assembled gold nanoparticles polymeric network blocks modular highly sensitive electrochemical biosensors for protein kinase activity analysis and inhibition. Anal Chem. 2014;86(12):6153-6159.

135. Jing X, Cao X, Wang L, et al. DNA-AuNPs based signal amplification for highly sensitive detection of DNA methylation, methyltransferase activity and inhibitor screening. Biosens Bioelectron. 2014;58:40-47.

136. Liang $\mathrm{H}$, Zhang $\mathrm{XB}, \mathrm{Lv} \mathrm{Y}$, et al. Functional DNA-Containing Nanomaterials: Cellular Applications in Biosensing, Imaging, and Targeted Therapy. Acc Chem Res. 2014;47(6):1891-1901.

137. Lee DE, Koo H, Sun IC, et al. Multifunctional nanoparticles for multimodal imaging and theragnosis. Chem Soc Rev. 2012;41(7):26562672 .

138. Li Z, Cheng E, Huang W, et al. Improving the yield of mono-DNAfunctionalized gold nanoparticles through dual steric hindrance. $J \mathrm{Am}$ Chem Soc. 2011;133(39):15284-15287.

139. Hallouard F, Anton N, Choquet P, et al. Iodinated blood pool contras media for preclinical X-ray imaging applications-a review. Biomaterials. 2010;31(24):6249-6268

140. Kim D, Park S, Lee JH, et al. Antibiofouling polymer-coated gold nanoparticles as a contrast agent for in vivo X-ray computed tomography imaging. J Am Chem Soc. 2007;129():7661-7665.

141. Liu Y, Ai K, Liu J, et al. A high-performance ytterbium-based nanoparticulate contrast agent for in vivo X-ray computed tomography imaging. Angew Chem Int Ed Engl. 2012;51(6):1437-1442. 
142. McQuaid HN, Muir MF, Taggart LE, et al. Imaging and radiation effects of gold nanoparticles in tumour cells. Sci Rep. 2016;6:19442.

143. Hainfeld JF, Smilowitz DN, Slatkin HM. The use of gold nanoparticles to enhance radiotherapy in mice. Phys Med Biol. 2004;49(18):N309N315.

144. Peng C, Wang H, Guo R, et al. Acetylation of dendrimer-entrapped gold nanoparticles: Synthesis, stability, and X-ray attenuation properties. $J$ Appl Polym Sci. 2011;119(3):1673-1682.

145. Peng C, Zheng L, Chen Q, et al. PEGylated dendrimer-entrapped gold nanoparticles for in vivo blood pool and tumor imaging by computed tomography. Biomaterials. 2011;33(4):1107-1119.

146. Peng C, Li K, Cao X, et al. Facile formation of dendrimer-stabilized gold nanoparticles modified with diatrizoic acid for enhanced computed tomography imaging applications. Nanoscale. 2012;4(21):6768-6778.

147. Li C, Chen T, Ocsoy I, et al. Gold-Coated $\mathrm{Fe}_{3} \mathrm{O}_{4}$ Nanoroses with Five Unique Functions for Cancer Cell Targeting, Imaging, and Therapy. Adv Funct Mater. 2014;24(12):1772-1780.

148. Zhang Y, Wen S, Zhao L, et al. Ultrastable polyethyleneimine-stabilized gold nanoparticles modified with polyethylene glycol for blood pool, lymph node and tumor CT imaging. Nanoscale. 2016;8(10):5567-5577.

149. Wen S, Li K, Cai H, et al. Multifunctional dendrimer-entrapped gold nanoparticles for dual mode CT/MR imaging applications. Biomaterials. 2013;34(5):1570-1580.

150. Zhang XD, Chen J, Luo Z, et al. Enhanced Tumor Accumulation of Sub$2 \mathrm{~nm}$ Gold Nanoclusters for Cancer Radiation Therapy. Adv Healthcare Mater. 2014;3(1):133-141.

151. Huang P, Bao L, Zhang C, et al. Folic acid-conjugated silica-modified gold nanorods for $\mathrm{X}$-ray/CT imaging-guided dual-mode radiation and photo-thermal therapy. Biomaterials. 2011;32(36):9796-9809.

152. Lee N, Choi SH, Hyeon T. Nano-sized CT contrast agents. Adv Mater. 2013;25(19):2641-2660.

153. Dou Y, Guo Y, Li X, et al. Size-Tuning Ionization To Optimize Gold Nanoparticles for Simultaneous Enhanced CT Imaging and Radiotherapy. ACS Nano. 2016;10(2):2536-2548.

154. Hainfeld JF, Slatkin DN, Focella TM, et al. Gold nanoparticles: a new X-ray contrast agent. Br J Radiol. 2006;79(939):248-253.

155. Rand D, Oritz V, Liu Y, et al. Nanomaterials for X-ray Imaging: Gold Nanoparticle Enhancement of X-ray Scatter Imaging of Hepatocellular Carcinoma. Nano Lett. 2011;11(7):2678-2683.

156. Chien CC, Chen HH, Lai SF, et al. Gold nanoparticles as high-resolution $\mathrm{X}$-ray imaging contrast agents for the analysis of tumor-related microvasculature. J Nanobiotechnol. 2012;10:10.

157. Kim CK, Ghosh P, Rotello V. Multimodal drug delivery using gold nanoparticles. Nanoscale. 2009;1(1):61-67.

158. Giljohann DA, Seferos DS, Daniel WL, et al. Gold nanoparticles for biology and medicine. Angew Chem Int Ed Engl. 2010;49(19):3280 3294 .
159. Lin J, Zhang H, Chen Z, et al. Penetration of lipid membranes by gold nanoparticles: insights into cellular uptake, cytotoxicity, and their relationship. ACS Nano. 2010;4(9):5421-5429.

160. Oh E, Delehanty J, Sapsford K, et al. Cellular uptake and fate of PEGylated gold nanoparticles is dependent on both cell-penetration peptides and particle size. ACS Nano. 2011;5(8):6434-6448.

161. Bartczak D, Nitti S, Millar TM, et al. Exocytosis of peptide functionalized gold nanoparticles in endothelial cells. Nanoscale. 2012;4(15):4470 4472 .

162. Marchesano V, Hernandez Y, Salvenmoser W, et al. Imaging inward and outward trafficking of gold nanoparticles in whole animals. ACS Nano. $2013 ; 7(3): 2431-2442$.

163. Yigit MV, Zhu L, Ifediba MA, et al. Noninvasive MRI-SERS Imaging in Living Mice Using an Innately Bimodal Nanomaterial. ACS Nano. 2011;5(2):1056-1066.

164. Kong KV, Lam Z, Goh WD, et al. Metal carbonyl-gold nanoparticle conjugates for live-cell SERS imaging. Angew Chem Int Ed Engl. 2012;51(39):9796-9799.

165. Black KC, Wang Y, Luehmann HP, et al. Radioactive 198Au-doped nanostructures with different shapes for in vivo analyses of their biodistribution, tumor uptake, and intratumoral distribution. ACS Nano. 2013;8(5):4385-4394.

166. Kircher MF, Zerda A, Jokerst JV, et al. A brain tumor molecular imaging strategy using a new triple-modality MRI-photoacoustic-Raman nanoparticle. Nat Med. 2012;18(5):829-834.

167. Zavaleta CL, Garai E, Liu JTC, et al. A Raman-based endoscopic strategy for multiplexed molecular imaging. Proc Natl Acad Sci. 2013;110(25):E2288-E2297.

168. Oh JW, Lim DK, Kim GH, et al. Thiolated DNA-Based Chemistry and Control in the Structure and Optical Properties of Plasmonic Nanoparticles with Ultrasmall Interior Nanogap. $J$ Am Chem Soc. 2014;136(40):14052-14059.

169. Tian L, Tadepalli S, Fei M, et al. Off-Resonant Gold Superstructures as Ultra bright Minimally Invasive Surface-Enhanced Raman Scattering (SERS) Probes. Chem Mater. 2015;27(16):5678-5684.

170. Srivastava S, Santos A, Critchley K, et al. Light-controlled selfassembly of semiconductor nanoparticles into twisted ribbons. Science. 2010;327(5971):1355-1359.

171. Peilong W, Zhenyu L, Xiaoou S, et al. Application of Au based nanomaterials in analytical science. Nano Today. 2017;12:64-97.

172. Wu DY, Li JF, Ren B, et al. Electrochemical surface-enhanced Raman spectroscopy of nanostructures. Chem Soc Rev. 2008;37(5):1025-1041.

173. Shang L, Dong SJ, Ulrich Nienhaus G. Ultra-small fluorescent metal nanoclusters: Synthesis and biological applications. Nano Today. 2011;6(4):401-418. 